\title{
CXCL17 is a Prognostic Biomarker That Distinguishes Severe Pandemic InfluenzaA(H1N1) from COVID-19
}

José Alberto Choreño-Parra

INER: Instituto Nacional de Enfermedades Respiratorias

Luis Armando Jiménez-Álvarez

INER: Instituto Nacional de Enfermedades Respiratorias

Gustavo Ramírez-Martínez

INER: Instituto Nacional de Enfermedades Respiratorias

Montserrat Sandoval-Vega

INER: Instituto Nacional de Enfermedades Respiratorias

Citlaltepetl Salinas-Lara

INNN: Instituto Nacional de Neurologia y Neurocirugia Manuel Velasco Suarez

Carlos Sánchez-Garibay

INNN: Instituto Nacional de Neurologia y Neurocirugia Manuel Velasco Suarez

César Luna-Rivero

INER: Instituto Nacional de Enfermedades Respiratorias

Erika M. Hernández-Montiel

INER: Instituto Nacional de Enfermedades Respiratorias

Luis Alejandro Fernández-López

INER: Instituto Nacional de Enfermedades Respiratorias

María Fernanda Cabrera-Cornejo

INER: Instituto Nacional de Enfermedades Respiratorias

Eduardo M. Choreño-Parra

UNAM: Universidad Nacional Autonoma de Mexico

Alfredo Cruz-Lagunas

INER: Instituto Nacional de Enfermedades Respiratorias

Andrea Domínguez

INER: Instituto Nacional de Enfermedades Respiratorias

Eduardo Márquez-García

INER: Instituto Nacional de Enfermedades Respiratorias

Carlos Cabello-Gutierrez

INER: Instituto Nacional de Enfermedades Respiratorias

Francina Valezka Bolaños-Morales

INER: Instituto Nacional de Enfermedades Respiratorias

Lourdes Mena-Hernández

INCMNSZ: Instituto Nacional de Ciencias Medicas y Nutricion Salvador Zubiran

Diego Delgado-Zaldivar

INCMNSZ: Instituto Nacional de Ciencias Medicas y Nutricion Salvador Zubiran

Daniel Rebolledo-García

INCMNSZ: Instituto Nacional de Ciencias Medicas y Nutricion Salvador Zubiran

Parménides Guadarrama-Ortiz

Centro Especializado en Neurocirugía y Neurociencias México

Nora E. Regino-Zamarripa

INER: Instituto Nacional de Enfermedades Respiratorias

Criselda Mendoza-Milla

INER: Instituto Nacional de Enfermedades Respiratorias

Ethel A. García-Latorre

Page $1 / 29$ 
IPN: Instituto Politecnico Nacional

Tatiana Sofía Rodríguez-Reyna

INCMNSZ: Instituto Nacional de Ciencias Medicas y Nutricion Salvador Zubiran

\section{Carmen M. Hernández-Cárdenas}

INER: Instituto Nacional de Enfermedades Respiratorias

\section{Shabaana A. Khader}

WUSTL: Washington University in St Louis

Albert Zlotnik

UCl: University of California Irvine

Joaquín Zúñiga ( $\square$ joazu@yahoo.com)

INER: Instituto Nacional de Enfermedades Respiratorias

\section{Research}

Keywords: influenza A(H1N1), SARS-CoV-2, COVID-19, pneumonia, chemokines, CXCL17

Posted Date: November 23rd, 2020

DOI: https://doi.org/10.21203/rs.3.rs-111860/v1

License: (c) (i) This work is licensed under a Creative Commons Attribution 4.0 International License. Read Full License 


\section{Abstract}

\section{Background}

CXCL17 is chemotactic for myeloid cells, exhibits broad-spectrum bactericidal activity, and is expressed in mucosal tissues. This chemokine is constitutively expressed in the respiratory tract, suggesting a role for CXCL17 in lung defenses. However, little is known about the possible participation of CXCL17 during respiratory infections in humans. Here, we evaluated the role of CXCL17 as a biomarker in patients with severe pandemic influenza A(H1N1) and coronavirus disease 2019 (COVID-19).

Methods

We conducted a prospective cohort study in hospitalized patients with severe influenza A(H1N1) and COVID-19 admitted to two national reference centers in Mexico City. Peripheral blood samples were obtained on admission for determinations of the serum levels of CXCL17 by enzyme-linked immunosorbent assay (ELISA). The expression of CXCL17 in lung autopsy specimens from patients that succumbed to both diseases was assessed by immunohistochemistry (IHQ). Serum CXCL17 levels were compared between patients grouped according to their disease and clinical outcome. The diagnostic and predictive value of serum CXCL17 levels was evaluated using machine-learning algorithms and regression analyses.

Results

A total of 92 patients were enrolled in the study, from which 68 were infected with influenza and 24 had COVID-19. Their clinical characteristics were similar, although dyspnea, rhinorrhea, and sputum production were more common during influenza, whereas dry cough and vomit were more frequent among COVID-19 patients. Both diseases induced the local expression of CXCL17 in the lung. However, serum levels of CXCL17 were increased only in patients with influenza but not COVID-19. CXCL17 not only differentiates influenza from COVID-19 but serves as a prognostic biomarker associated with mortality and renal failure in influenza patients. Using cell culture assays, we also identified that human alveolar A549 cells and peripheral blood monocyte-derived macrophages produce CXCL17 after influenza A(H1N1) pdm09 virus infection.

Conclusions

Our results suggest a possible role for CXCL17 in the pathogenesis of influenza $A(H 1 N 1)$, supporting the use of this molecule as a prognostic biomarker. Future studies on the role of CXCL17 in COVID-19 are warranted.

\section{Background}

During the upcoming flu season, the world will face one of the most challenging public health crises in recent history due to the convergence of influenza and coronavirus disease 2019 (COVID-19). Both entities have the potential to cause severe disease manifestations, mainly associated with an exacerbated immune activation [1, 2]. Despite the significant progress in understanding the immunopathology of influenza and COVID-19, the role of many immune mediators is as yet unknown. Furthermore, immunological features that may differentiate the two diseases are not well characterized.

The C-X-C motif chemokine ligand 17 (CXCL17) is a mucosal chemokine expressed in the respiratory tract under both homeostatic and inflammatory conditions [3-5]. Its homeostatic functions include the recruitment of various myeloid cell populations (dendritic cells (DCs), monocytes, and macrophages) to mucosal tissues, including the lung [6]. Under inflammatory conditions, its functions are less well characterized, although it has been reported to promote anti-inflammatory activities and likely prevent autoimmunity $[4,6]$. CXCL17 also exhibits broad antimicrobial activity $[4,6]$, suggesting that it may play an important role in protective immunity against respiratory pathogens. In viral infections, there is only a report suggesting that CXCL17 participates in immunity against genito-urinary herpes infections [7]. However, despite CXCL17 being one of the most highly expressed chemokines in the human lung, its potential role in human lung viral infections has not been studied.

Here, we report the high levels of CXCL17 in serum from patients with acute respiratory distress syndrome (ARDS) associated with pandemic influenza $A(H 1 N 1)$. Conversely, CXCL17 serum levels in COVID-19 patients were not elevated, indicating that CXCL17 serum levels are a diagnostic marker that can differentiate individuals with influenza $A(H 1 N 1)$ infection from COVID-19. We also analyzed the pattern of expression of this chemokine in lung specimens from patients with influenza and SARS-CoV-2 infection. Importantly, analyses of CXCL17 serum levels in influenza A (H1N1) patients revealed that high levels of CXCL17 correlated with poor prognosis, including kidney injury and death. Finally, we identified that human alveolar A549 cells and peripheral blood monocyte-derived macrophages produce CXCL17 in 
response to influenza $A(\mathrm{H} 1 \mathrm{~N} 1)$ pdm09 virus infection. Taken together, our results support a role for CXCL17 in the pathogenesis of severe viral respiratory infections in humans.

\title{
Materials And Methods
}

\author{
Study design and participants
}

We conducted a cohort study in hospitalized patients ( $N=68)$ with laboratory-confirmed influenza $A(H 1 N 1)$ pdm09 virus infection (hereinafter referred to as influenza) that attended the emergency department of the National Institute for Respiratory Diseases (INER) of Mexico during the 2018/19 and 2019/20 flu seasons. Patients with influenza-like illness (ILI) that progressed to ARDS requiring mechanical ventilation (MV) and admission to the intensive care unit (ICU) were eligible. These subjects were screened for influenza using the rapid influenza diagnostic test (RIDT) Fuji dri-chem immuno AG cartridge FluAB kit (Fujifilm Corp, Tokyo, Japan) in fresh respiratory swab specimens. Simultaneously, further molecular characterization of the causative influenza virus subtype was assessed by RT-PCR, as previously described [8].

In addition, we included a group of ( $\mathrm{N}=24)$ patients with COVID-19 that attended INER or the National Institute of Medical Sciences and Nutrition (INCMNSZ) in Mexico City, from March to May of 2020. The infection with SARS-CoV-2 was detected by RT-PCR, as described below [9]. None of the patients enrolled in the study had human immunodeficiency virus (HIV) infection. We retrieved clinical and demographic data from the participants. Peripheral blood samples were taken from each patient within the first $24 \mathrm{~h}$ following hospital admission to determine serum CXCL17 levels. An additional blood sample was obtained from patients with influenza seven days after admission. Healthy volunteer individuals $(\mathrm{N}=30)$ were also recruited to participate in the study and served as controls.

\section{RT-PCR for SARS-CoV-2 detection}

Briefly, viral RNA was extracted from clinical samples with the MagNA Pure 96 system (Roche, Penzberg, Germany). The RT-PCR reactions were performed in a total volume of $25 \mu \mathrm{L}$, containing $5 \mu \mathrm{L}$ of RNA, $12.5 \mu \mathrm{L}$ of $2 \times$ reaction buffer provided with the Superscript III one-step RTPCR system with Platinum Taq Polymerase (Invitrogen, Darmstadt, Germany; containing 0.4 mM of each deoxyribose triphosphates (dNTP) and $3.2 \mathrm{mM}$ magnesium sulfate), $1 \mu \mathrm{L}$ of reverse transcriptase/ Taq mixture from the kit, $0.4 \mu \mathrm{L}$ of a $50 \mathrm{mM}$ magnesium sulfate solution (Invitrogen), and $1 \mu \mathrm{g}$ of nonacetylated bovine serum albumin (Roche). Primer and probe sequences, as well as optimized concentrations, are shown in Supplemental Table 1. All oligonucleotides were synthesized and provided by Tib-Molbiol (Berlin, Germany). Thermal cycling was performed at $55^{\circ} \mathrm{C}$ for $10 \mathrm{~min}$ for reverse transcription, followed by $95^{\circ} \mathrm{C}$ for $3 \mathrm{~min}$ and then $45 \mathrm{cycles}$ of $95^{\circ} \mathrm{C}$ for $15 \mathrm{~s}, 58^{\circ} \mathrm{C}$ for $30 \mathrm{~s}$.

In vitro infection assays with influenza $A(H 1 N 1)$ pdm09 virus

Influenza A(H1N1) pdm09 virus was isolated from patients with severe pneumonia in Madin-Darby canine kidney cells (MDCK). Virus infectivity was assessed by titration of tissue culture infection dose $50 \%\left(\mathrm{TCID}_{50}\right)$ in MDCK cells, as previously described [10]. Human lung adenocarcinoma epithelial cells A549 were purchased from the American Type Culture Collection (ATCC, Rockville, MD) and cultured in DMEM with $10 \%$ fetal bovine serum (Lonza) at $37{ }^{\circ} \mathrm{C}$ and $5 \% \mathrm{CO} 2$. Human macrophages were obtained and cultured as described before [10]. A549 epithelial cells and macrophages were infected with influenza A(H1N1) pdm09 virus at a multiplicity of infection (MOI) of 5 for one hour. Mock-treated cells received a virus-free culture medium. After incubation, cells were washed twice with phosphate buffer saline (PBS). Media containing the influenza virus was replaced with a virus-free culture medium. Supernatants were collected after 24,48 , and 72h, for CXCL17 quantitation.

\section{CXCL 17 levels}

Levels of human CXCL17 in serum and culture supernatants were determined by enzyme-linked immunosorbent assay (ELISA) using a commercial kit (MBS916471, My BioSource, USA) following the manufacturer's instructions.

\section{Histological analysis and immunohistochemistry}

Formalin-fixed and paraffin-embedded lung autopsy specimens from patients who died of influenza or COVID-19 (N=2 patients per group) were obtained from the Pathology Department of the INER. Sections of 3-5 $\mu$ m were processed for hematoxylin-eosin (H\&E) staining for histopathological analysis. For immunohistochemistry (IHQ), lung sections were mounted on silane-covered slides, deparaffinized, and the endogenous peroxidase blocked. Sections were incubated overnight at room temperature with an optimal dilution (1:100) of the Mouse AntiHuman CXCL17/VCC-1 Monoclonal Antibody (Clone \# 422208, MAB4207, R\&D Systems, Minneapolis, MN). Secondary biotinylated antibodies labeled with peroxidase were added, and those attached were revealed with diaminobenzidine (ImmunoCruz ${ }^{\mathrm{TM}}$ rabbit $^{\mathrm{ABC}}$ Staining System, Santa Cruz Biotechnology, Santa Cruz, CA). Slides were counter-stained with hematoxylin. 
All enrolled individuals or their legal guardians provided written consent to participate in the study. Serum samples were managed according to the Mexican law NOM-012-SSA3-2012, which establishes the criteria for the execution of clinical investigations in humans. The Ethics Committee of INER and INCMNSZ in Mexico City approved the study.

\section{Statistical analysis}

Descriptive statistics were used to characterize the population under study clinically. Frequencies and proportions were calculated for categorical data. Means, medians, standard deviations (SD), interquartile ranges (IQR), and 95\% confidence intervals (CI) were used for continuous variables. Differences in categorical variables between groups were assessed by the Fisher exact or Chi-square test, as appropriate. For comparisons of continuous variables between two groups, we used the Mann-Whitney $U$ test. For differences of continuous data between more than two groups, we used the Kruskal Wallis test with post hoc Dunn test. Differences in serum levels of CXCL17 measured in serial samples were determined using the Wilcoxon matched-pairs signed-rank test. Multiple linear regression analyses using Spearman rank correlation coefficients were used to determine correlations between continuous clinical variables and serum levels of CXCL17. The K-means algorithm was used for clustering study participant characteristics according to their diagnosis (influenza or COVID19 ) and clinical outcome (survival or fatality). Before data visualization, clinical features and laboratory parameters were scaled and centered.

To identify the variables with the highest impact on disease diagnosis (influenza, COVID-19) and adverse outcomes (death), random forest analyses of 500 classification and regression trees (CARTs) were performed. The diagnostic accuracy of serum CXCL17 levels and other selected variables identified by random forest for was further evaluated by bivariate logistic regression and Receiver Operating Characteristic (ROC) curve (AUC) analyses. In addition, Kaplan-Meier curves were constructed to look for differences in survival according to serum CXCL17 levels dichotomized by the ROC curve threshold with the highest diagnostic accuracy estimated using the Youden index. For random forest and logistic regression analyses, patients with any missing value in the variables of interest were excluded.

All analyses were conducted using GraphPad Prism 8 (La Jolla, CA) and R Statistical Software (Foundation for Statistical Computing, Vienna, Austria). Specific analytical tests are also described in the figure legends. Values of $p \leq 0.05$ were considered as significant: ${ }^{\star} p \leq 0.05$, ${ }^{* *} p \leq 0.01,{ }^{* * *} p \leq 0.001, * * * * p \leq 0.0001$.

\section{Results}

\section{Participant characteristics}

A total of 92 patients were enrolled in the study, from which 68 were infected with influenza and 24 had COVID-19. The clinical characteristics of the study participants are summarized in Table 1. Seventy percent of influenza patients were male, with a median age of 48 years. Similarly, COVID-19 affected males (75\%) preferentially, with a median age of 50 years. Obesity was more common among influenza patients, while the prevalence of other comorbidities did not differ between both diseases. Cough, dyspnea, fever, myalgia, and arthralgia were the most frequent symptoms of respiratory illness in both participant groups. Interestingly, dyspnea, rhinorrhea, and sputum production were more common during influenza, whereas dry cough and vomit were more frequent among COVID-19 patients (Table 1).

Triage vital signs were similar between groups, except for a higher blood temperature and heart rate in influenza patients. Also, most laboratory parameters routinely tested in emergency departments did not differ between individuals with influenza and COVID-19. The levels of aspartate aminotransferase (AST), lactate dehydrogenase (LDH), alkaline phosphatase (ALP), and procalcitonin were increased in influenza patients as compared to COVID-19 subjects (Table 1). Similarly, severity of illness scores at admission, including the Sequential Organ Failure Assessment (SOFA), and the Acute Physiology And Chronic Health Evaluation II (APACHE-II), were higher in influenza cases. We should note that despite this, COVID-19 patients showed higher mortality compared to influenza $(41 \%$ vs. $23 \%, p=0.1151)$.

\section{Expression of CXCL 17 in the lung of patients with influenza or COVID-19}

CXCL17 is constitutively expressed in the respiratory tract and lungs of mice and humans [3, 4]. Under inflammatory conditions, the production of this chemokine is known to be further upregulated $[4,6,7,11,12]$. To investigate whether CXCL17 may participate in immunity against influenza $\mathrm{A}(\mathrm{H} 1 \mathrm{~N} 1)$ and SARS-CoV-2, we analyzed the tissue expression pattern of CXCL17 in lung autopsy specimens obtained from patients who died of influenza or COVID-19. The histological changes induced in the lungs during influenza were mainly characterized by intra-alveolar inflammatory infiltrates consisting of macrophages and polymorphonuclear cells scattered between areas of edema, hemorrhage, and fibrin deposits (Figure 1a, left upper panel). We also noted that the integrity of alveolar walls and the micro-architecture of the lung were conserved in influenza patients (Supplemental Figure 1). CXCL17 expression was detected mainly within the cytoplasm of 
infiltrating macrophages, but not in polymorphonuclear cells. The expression of CXCL17 was also detected within alveolar epithelial cells (Figure 1a, left lower panel). Blood vessels and pleura did not express CXCL17 in lung autopsy specimens from influenza patients (data not shown).

Meanwhile, SARS-CoV-2 induced distinctive morphological changes in the infected lung, characterized by an intense inflammatory infiltrate affecting extensive areas of the parenchyma, as well as the thickness of alveolar walls, and partial loss of the histological architecture of the the lung (Supplemental Figure 1 and Figure 1a, right upper panel). These changes are compatible with interstitial pneumonia. As observed in influenza infected lung sections, the expression of CXCL17 in the lungs of deceased COVID-19 patients was also detected within macrophages and alveolar epithelial cells (Figure 1a, right lower panel). CXCL17 was not expressed in endothelial cells of alveolar capillaries, but pleura showed increased CXCL17 expression (data not shown). Collectively, these findings indicate that influenza and SARSCoV-2 promote the production of CXCL17 in the infected lung.

High serum levels of CXCL17 distinguish influenza from COVID-19

Although CXCL17 is mainly produced at mucosal surfaces, increased serum levels of this chemokine might serve as a readout of active local immune responses. Thus, we addressed whether the CXCL17-mediated responses found in the lungs of influenza- and SARS-CoV-2-infected patients could be also detected in the serum. Our results indicate that CXCL17 levels were significatively elevated in the serum of influenza cases, but not in healthy donors (HD) or COVID-19 subjects. The latter group indeed showed low serum CXCL17 levels, similar to the levels observed in HD (Figure 1b). These findings contrast with the expression of CXCL17 detected in the lung of COVID-19 patients, suggesting that the levels of CXCL17 in serum and its expression in lung tissue specimens do not correlate during the course of the disease. Nonetheles, our lung immunohistochemical analyses only focused on the expression of this chemokine in the late stages of influenza and COVID-19, whereas serum samples were taken within the first day after patients' hospital admission.

Given that these differences suggested distinctive serum CXCL17 responses during influenza and COVID-19, we next investigated if CXCL17, along with other clinical characteristics, could have certain diagnostic value to discriminate between both viral infections. In an unsupervised clutering analysis, we found that some influenza patients grouped together according to their clinical and laboratory parameters, but another cluster was formed by combined influenza and COVID-19 subjects (Figure 1c). This suggests that the differentiation of the two infections by clinical characteristics would be difficult. Nonetheless, in a random forest analysis, CXCL17 was among the most explicative variables of the viral subtype. Indeed, in a bivariate logistic regression analysis using the variables identified in the random forest algorithm, only CXCL17, along with procalcitonin, showed significant association with influenza (Supplemental Figure 2). LDH and ALP were marginally associated with influenza, whereas platelets showed no correlation with any type of infection. Interestingly, although irrelevant in the random forest algorithm, symptoms such as dyspnea, rhinorrhea, and sputum production were predictors of influenza, whereas dry cough and vomit were associated with COVID-19.

To further estimate the diagnostic value of CXCL17, we performed a ROC curve analysis with the serum levels of CXCL17 of influenza and COVID-19 subjects. We observed that CXCL17 levels could reliably differentiate between influenza and COVID-19, with an AUC of 0.81 (Figure 1e). Using a cut-off value of $841 \mathrm{pg} / \mathrm{mL}$, elevated serum levels of this chemokine have a $78.2 \%$ sensitivity, $73.5 \%$ specificity, $89.2 \%$ PPV, $51.3 \% \mathrm{NPV}$, and an OR of $8.79(3.1-26,95 \% \mathrm{Cl})$ to distinguish influenza from COVID-19. Together, our results point to the diagnostic use of serum CXCL17 levels in patients with acute respiratory illness to enable distinguishing infection between these viruses.

\section{Dynamics and prognostic value of serum CXCL17 levels in influenza}

Next, we evaluated the dynamics of serum CXCL17 levels during influenza. For this purpose, we grouped influenza patients according to the duration of their illness, defined as the period from symptom onset to hospital admission. Interestingly, we found that levels of CXCL17 increased early in influenza patients within the first two days following the onset of symptoms, and levels remained increased during the first two weeks of illness. However, the maximum levels of CXCL17 were observed among influenza patients seeking medical attention three weeks after the onset of symptoms (Figure 2a). In 54 of the 68 influenza patients enrolled in the study, an additional serum sample was obtained seven days (D7) following hospital admission (D0). Although there were no differences in CXCL17 between D0 and D7, most patients showed constant or decreasing chemokine levels (Figure 2b), except for one individual who showed a notable increase in CXCL17 and succumbed to the infection. Overall, the dynamics of CXCL17 levels post-hospital admission were similar in survivors and deceased influenza patients (Figure 2c).

Importantly, serum levels of CXCL17 at D0 were significatively higher in patients who died of influenza as compared to survivors (Figure 2d). Indeed, CXCL17 again was among the variables with the higher importance for influenza-associated mortality in a random forest analysis

(Figure 2e), showing an AUC of 0.70 to differentiate both groups in the ROC curve analysis (Figure 2f). Serum levels of CXCL17 above $1,128 \mathrm{pg} / \mathrm{mL}$ predicted a fatal outcome with a $75 \%$ sensitivity and $50 \%$ specificity, showing a non-significant OR value for mortality of 3.0 
(0.86-9.24, 95\% Cl). The survival of patients with CXCL17 below such cut-off value was lower at day 14 after admission when compared to individuals with higher levels of this chemokine (Figure $2 \mathrm{~g}$, Table 2). Conversely, accumulated survival at days 28 and 60 following hospital admission was lower in influenza patients with serum levels of CXCL17 $\geq 1,128 \mathrm{pg} / \mathrm{mL}$.

Most clinical and laboratory parameters did not differ between the two groups of influenza patients (Supplemental Table 2). Furthermore, none of these clinical characteristics impacted the serum levels of CXCL17 (Figure 3a), and this chemokine was not associated with the severity of ARDS in terms of the PaO2/FiO2 values at admission (Figure $3 \mathbf{b}$ ). These results together suggest that CXCL17 represents an independent prognostic factor of mortality in influenza. In contrast, no differences in serum CXCL17 levels at admission were observed between survivor- and deceased-COVID-19 patients (Figure 3c).

We also noted that CXCL17 was increased among influenza patients that developed acute kidney injury (AKIN; Figure 3d), and its levels were even higher in individuals requiring renal replacement therapy (Figure $3 \mathbf{e}$ ). Using the same cut-off value of 1,128pg/mL, elevated serum levels of CXCL17 showed a 73.6\% sensitivity, 51\% specificity, and a non-significant OR of 2.91 (0.96-8.28, 95\% Cl) to predict the development of AKIN (Figure 3f). Similarly, increased levels of CXCL17 showed an 87.5\% sensitivity, 53.8\% specificity, and a significant OR of 8.16 (1.71$38,95 \% \mathrm{Cl}$ ) to predict the need for renal replacement therapy (Figure $\mathbf{3 g}$ ).

\section{Influenza $A(H 1 N 1)$ pdm09 virus induces the production of CXCL 17 in human lung epithelial cells and macrophages}

The higher levels of CXCL17 in sera from influenza patients prompted us to investigate the possible cellular sources of CXCL17 during influenza. To this end, we infected human A549 lung alveolar epithelial cells and peripheral blood monocyte-derived macrophages with a clinical isolate of the influenza $\mathrm{A}(\mathrm{H} 1 \mathrm{~N} 1)$ pdm09 virus. Interestingly, while both human cell types produced high amounts of $\mathrm{CXCL} 17$ at $24 \mathrm{~h}$, $48 \mathrm{~h}$, and $72 \mathrm{~h}$ after the infection, A549 epithelial cells produced lower levels of CXCL17 in response to influenza as compared to macrophages (Figure 3h-i). These findings are cosnsitent with the the expression of CXCL17 in lung macrophages and alveolar epithelial cells in autopsy specimens from influenza patients. This demonstrates that the influenza A(H1N1) pdm09 virus stimulates CXCL17 expression in humans, both in vivo and in vitro.

\section{Discussion}

The clinical spectrum of ARDS encompasses very severe forms of the disease characterized by a profound respiratory failure. These manifestations are often related to dysregulated immune reactions elicited by different insults. The quality and magnitude of such responses may vary according to the causative agent of the illness. Thus, particular immune profiles of local and circulating cytokines may serve as readouts to differentiate between specific causes of ARDS. Here, we demonstrate that the CXCL17 expression induced in human lungs during influenza and COVID-19 are different. These differences include distinctive serum levels of CXCL17 duirng influenza but not COVID-19. Chemokines can be divided into homeostatic or inflammatory depending on their expression patterns [13]. CXCL17 is considered a 'dual' chemokine because it exhibits characteristics of both homeostatic and inflammatory chemokines. It is constitutively expressed in the mucosa of the respiratory tract with potential functions in lung defenses [14] and is known to chemoattract macrophages [6]. This chemokine was the last member of the C-X-C motif chemokine ligand family to be reported [3], and as such, its role during respiratory inflammation or infections is not well understood.

We should note that bioinformatics analyses of public gene expression databases indicate that CXCL17 is the highest expressed chemokine in the normal trachea and bronchus and among the highest expressed chemokines in the normal human lung [4]. These observations strongly suggest important homeostatic functions for CXCL17 in the respiratory tract. Previous studies have shown that CXCL17 is strongly upregulated in idiopathic pulmonary fibrosis [4]. This, along with data from our current study indicate that CXCL17 is very likely to have important functions in the pathogenesis of inflammatory/infectious diseases of the lung as well. Past studies have provided indirect evidence about a possible role of CXCL17 in immunity against respiratory infections. For instance, it has been shown that CXCL17 has a potent bactericidal activity over bacteria causative of respiratory infections [4]. The expression of the CXCL $17 \mathrm{gene}$ was also found upregulated in group 3 innate lymphoid cells isolated from lung tissues of patients with tuberculosis (TB) [15], suggesting a role for CXCL17 against Mycobacterium tuberculosis (Mtb) infection. However, CXCL17 plays redundant activities during anti-Mtb immunity in murine models [16]. In this context, our study represents the first direct evaluation of the role of CXCL17 during respiratory infections in humans.

Our results indicate that the influenza $\mathrm{A}(\mathrm{H} 1 \mathrm{~N} 1)$ pdm09 virus and SARS-CoV-2 stimulate the local production of $\mathrm{CXCL} 17$ in the lungs. This coincides with the high induction of CXCL17 in the BAL of COVID-19 identified by other investigators [17]. However, we found that the magnitude of CXCL17 expression in the serum was also robust during influenza but minimal in COVID-19 patients. These are contrasting observations, although we have to consider that the lung tissue specimens in our study were obtained post-mortem. Therefore, our immunohistochemical analysis evaluated the expression of CXCL17 only during the latest phases of viral infection, whereas serum chemokine determinations captured early responses against COVID-19 and influenza. Analysis of CXCL17 expression in lung biopsy

Page 7/29 
specimens obtained early during the infection would have clarified this point. Despite this, we speculate that lung and serum CXCL17 levels may increase as the infection with SARS-CoV-2 progresses in patients with severe disease. Whether longitudinal changes in serum CXCL17 levels could have a prognostic value in COVID-19 should be investigated in future studies.

Overall, our findings may indicate that SARS-CoV-2 and influenza A(H1N1) pdm09 trigger local and systemic innate immune mechanisms of distinctive features and dynamics. These variations may be also related to different abilities of influenza and SARS-CoV virus strains to induce local CXCL17 production and translocation to the blood according to their virulence. Indeed, previous research showed that the infection of human bronchial epithelial cells, human tracheobronchial epithelial cells, and human alveolar A549 cells with seasonal influenza $A(H 3 N 2)$ virus, promotes robust upregulation of $C X C L 17$. However, the infection with more virulent $A(H 5 N 1)$ and $A(H 7 N 9)$ influenza virus subtypes induces minor changes in the expression of CXCL17[18]. Similarly, in a yet unpublished experiment available from the Influenza Research Database (IRD), the infection of 2B-4cells/sorted Calu-3 cells with a wild type strain of the 2002-2003 SARS-CoV (icSARS CoV) did not stimulate strong expression of $C X C L 17$ [19]. In contrast, the infection with icSARS ExoNI and icSARS dNSP16, which are attenuated mutant strains of SARS-CoV $[3,6,20]$, upregulated the expression of CXCL 17.

Notably, we also demonstrated that these differences could be harnessed for clinical applications, as serum CXCL17 levels determined at hospital admission are useful to distinguish between influenza and COVID-19 in patients with ARDS. This is important, as it is highly likely that both diseases will converge during the upcoming flu season. During such a predicted scenario, the differentiation of influenza and COVID-19 by clinical characteristics may be complicated. Indeed, our results and previous studies show that only a few nonspecific symptoms and routine laboratory tests are useful for this diagnostic dilemma [21-23]. However, the discrimination of the causative pathogen has direct therapeutic implications, including the selection of the adequate anti-viral drug. Thus, novel biomarkers with high diagnostic value to distinguish influenza and COVID-19 are urgently needed.

Another striking finding of our study is that serum levels of CXCL17 impact on influenza-assocaited mortality. In this sense, it is known that several chemokines participate in the immune response against influenza viruses [24, 25]. Most of them are produced in high amounts and mediate pathology due to their pro-inflammatory properties [26, 27]. Our data indicate that the production of CXCL17 is also highly potentiated during the early stages of influenza, as the serum levels reported here are as high as those found in other inflammatory and human autoimmune disorders [18]. Moreover, we demonstrate that both macrophages and lung epithelial cells can become sources of CXCL17 after infection with the influenza $\mathrm{A}(\mathrm{H} 1 \mathrm{~N} 1)$ pdm09 virus.

CXCL17 normally mediates the recruitment of myeloid cells to the lung [6]. Cell subtypes responding to this chemokine include DCs, monocytes, and macrophages $[3,6,20]$. However, we should note that the nature of the myeloid cells recruited by CXCL17 in vivo has not been established in humans, although the fact that CXCL17 is a mucosal chemokine expressed only in the respiratory and digestive tracts suggests that it is recruiting unique population(s) of myeloid cells to mucosal tissues which remain to be functionally characterized. Increased and sustained recruitment of myeloid cells to the lungs is associated with immunopathology and mortality in influenza [24, 25]. Accordingly, our data indicate that influenza patients with higher CXCL17 levels have lower survival, suggesting a possible pathogenic role for this chemokine. We should also mention that CXCL17 also mediates anti-inflammatory activities [26, 27]. Thus, our findings may reflect a regulatory mechanism for the cytokine storm underlying severe influenza, via the production of high levels of anti-inflammatory mediators.

The role of innate immune cells responding to CXCL17 in COVID-19 is unknown, but recent studies have found that the numbers of circulating DCs and monocytes are reduced in patients with severe SARS-CoV-2 infection as compared to patients with milder forms of the disease [28]. In contrast, we found a high number of macrophages in the lungs of patients that died of COVID-19. Together, these data point to a possible role for chemokines involved in the recruitment of myeloid cells to the lungs during COVID-19, and CXCL17 is an excellent candidate to mediate this recruitment [6]. Finally, while we did not perform in vitro infections with SARS-CoV-2, we show that these cell types express CXCL17 during COVID-19 in vivo. However, whether CXCL17 plays a pathogenic or protective role during influenza or COVID-19 is not apparent from these data.

\section{Conclusions}

In conclusion, our study provides evidence supporting an important role for the chemokine CXCL17 in the pathogenesis of both influenza and COVID-19. Future studies in CXCL17 deficient animals are required to better understand the function of this chemokine in lung defenses against the influenza $\mathrm{A}(\mathrm{H} 1 \mathrm{~N} 1)$ pdm09 virus. Also, future studies addressing the possible role of CXCL17 in immunity against SARS-CoV-2 are warranted.

\section{Abbreviations}


95\% Cl, 95\% confidence interval; AKIN, acute kidney injury; ALP, alkaline phosphatase; ALT, alanine aminotransferase; APACHE-II, Acute Physiology and Chronic Health disease Classification System II; ARDS, acute respiratory distress syndrome; AST, aspartate aminotransferase; AUC, area under the ROC curve; BAL, bronchoalveolar lavage; BMI, body mass index; BUN, blood ureic nitrogen; CARTs, classification and regression trees; CO2, carbon dioxide; COPD, chronic obstructive pulmonary disease; COVID-19, coronavirus disease 2019; CPK, creatine phosphokinase; $\mathrm{Cr}$, creatinine; CXCL17, C-X-C motif chemokine ligand 17; DCs, dendritic cells; dNTP, deoxyribose triphosphates; ECMO, extra-corporeal membrane oxygenation; ELISA, enzyme-linked immunosorbent assay; H\&E, hematoxylin-eosin; HCO3, bicarbonate; HD, healthy donors; Hgb, hemoglobin; HIV, human immunodeficiency virus; ICU, intensive care unit; IHQ, immunohistochemistry; ILI, influenza-like illness; INCMNSZ, National Institute of Medical Sciences and Nutrition; INER, Instituto Nacional de Enfermedades Respiratorias Ismael Cosío Villegas; IQR, interquartile ranges; IRD, Influenza Research Database; LDH, lactate dehydrogenase; MAP, mean arterial pressure; MDCK, Madin-Darby canine kidney cells; MOI, multiplicity of infection; Mtb, Mycobacterium tuberculosis; MV, mechanical ventilation; NLR, neutrophil/lymphocyte ratio; OR, odds ratio; OSA, obstructive sleep apnea syndrome; PaO2, partial pressure of oxygen in arterial blood; PBS, phosphate buffer saline; PCO2, partial pressure of carbon dioxide in the blood; RIDT, rapid influenza diagnostic test; ROC, Receiver Operating Characteristic; RT-PCR, real-time polymerase chain reaction; SAH, systemic arterial hypertension; SARS-CoV-2, severe acute respiratory syndrome coronavirus 2; SD, standard deviations; SOFA, Sequential Organ Failure Assessment; TB, tuberculosis; TCID50, tissue culture infection dose $50 \%$.

\section{Declarations}

\section{Ethics approval and consent to participate}

The current study was reviewed and approved by the Institutional Review Boards of the Instituto Nacional de Enfermedades Respiratorias Ismael Cosío Villegas (INER, approval number: B28-16 and B09-20) and the National Institute of Medical Sciences and Nutrition (INCMNSZ, approval number: 3349) in Mexico City. All participants or their legal guardians provided written consent to participate in the study.

\section{Consent for publication}

All authors have approved the manuscript.

\section{Availability of data and material}

Clinical data of study participants are available from the corresponding author upon reasonable request.

\section{Competing interests}

The authors declare no competing interests.

\section{Funding}

Institutional research funds of INER supported the current study. This project also received funding from the National Council of Science and Technology of Mexico (CONACyT) under the research contracts: SECTEI/050/2020, Secretaría de Ciencia, Tecnología e Innovación de la Ciudad de México (SECTEI CDMX); FORDECYT/10SE/2020/05/14-06 and FORDECYT/10SE/2020/05/14-07 from the Fondo Institucional de Fomento Regional para el Desarrollo Científico y Tecnológico y de Innovación (FORDECYT). JC-P was supported by a scholarship (CVU 737347) from CONACyT to his Ph.D. degree. Funders did not play any role in the study design and conduction.

\section{Author contributions}

Collected clinical data and biological samples for the study: JC-P, MS-V, CH-C, EH-M, MC-C, NR-Z, CM-M, AC-L, AD, LM-H, DD-S, DR, and CM-M. Performed in vitro infections: GR-M, LJ-A, CC-G, EM-G, and AC-L. Performed CXCL17 ELISA assays: JC-P, LJ-A, GR-M, and LF-L. Obtained and analyzed autopsy lung specimens: CS-L, CS-G, FB-M, PG-O, and CL. Analyzed and discussed data: JC-P, E-CP, MS-V, EG-L, TR-R, SA-K, and JZ. Drafted the manuscript: JC-P, SA-K, AZ, and JZ. All authors read and approved the final version of the paper.

\section{Acknowledgments}

To all the patients that participated in the study and their family members.

\section{References}


1. Coperchini F, Chiovato L, Croce L, Magri F, Rotondi M: The cytokine storm in COVID-19: An overview of the involvement of the chemokine/chemokine-receptor system. Cytokine Growth Factor Rev 2020, 53:25-32.

2. Liu Q, Zhou YH, Yang ZQ: The cytokine storm of severe influenza and development of immunomodulatory therapy. Cell Mol Immunol 2016, 13(1):3-10.

3. Pisabarro MT, Leung B, Kwong M, Corpuz R, Frantz GD, Chiang N, Vandlen R, Diehl LJ, Skelton N, Kim HS et al: Cutting edge: novel human dendritic cell- and monocyte-attracting chemokine-like protein identified by fold recognition methods. $\mathrm{J} / \mathrm{mmuno} / 2006$, 176(4):2069-2073.

4. Burkhardt AM, Tai KP, Flores-Guiterrez JP, Vilches-Cisneros N, Kamdar K, Barbosa-Quintana O, Valle-Rios R, Hevezi PA, Zuniga J, Selman $\mathrm{M}$ et al: CXCL17 is a mucosal chemokine elevated in idiopathic pulmonary fibrosis that exhibits broad antimicrobial activity. $J$ Immunol 2012, 188(12):6399-6406.

5. Weinstein EJ, Head R, Griggs DW, Sun D, Evans RJ, Swearingen ML, Westlin MM, Mazzarella R: VCC-1, a novel chemokine, promotes tumor growth. Biochem Biophys Res Commun 2006, 350(1):74-81.

6. Burkhardt AM, Maravillas-Montero JL, Carnevale CD, Vilches-Cisneros N, Flores JP, Hevezi PA, Zlotnik A: CXCL17 is a major chemotactic factor for lung macrophages. J Immuno/ 2014, 193(3):1468-1474.

7. Srivastava R, Hernandez-Ruiz M, Khan AA, Fouladi MA, Kim GJ, Ly VT, Yamada T, Lam C, Sarain SAB, Boldbaatar U et al: CXCL17 Chemokine-Dependent Mobilization of CXCR8(+)CD8(+) Effector Memory and Tissue-Resident Memory T Cells in the Vaginal Mucosa Is Associated with Protection against Genital Herpes. J Immuno/ 2018, 200(8):2915-2926.

8. Castillejos M, Cabello-Gutiérrez C, Alberto Choreño-Parra J, Hernández V, Romo J, Hernández-Sánchez F, Martínez D, Hernández A, Jiménez-Álvarez L, Hernández-Cardenas CM et al: High performance of rapid influenza diagnostic test and variable effectiveness of influenza vaccines in Mexico. International Journal of Infectious Diseases 2019, 89:87-95.

9. Corman VM, Landt O, Kaiser M, Molenkamp R, Meijer A, Chu DK, Bleicker T, Brunink S, Schneider J, Schmidt ML et al: Detection of 2019 novel coronavirus (2019-nCoV) by real-time RT-PCR. Euro Surveill 2020, 25(3):2000045.

10. Ramírez-Martínez G, Cruz-Lagunas A, Jiménez-Alvarez L, Espinosa E, Ortíz-Quintero B, Santos-Mendoza T, Herrera MT, Canché-Pool E, Mendoza C, Bañales JL et al: Seasonal and pandemic influenza H1N1 viruses induce differential expression of SOCS-1 and RIG-I genes and cytokine/chemokine production in macrophages. Cytokine 2013, 62(1):151-159.

11. Hernandez-Ruiz M, Zlotnik A, Llorente L, Hernandez-Molina G: Markedly high salivary and lacrimal CXCL17 levels in primary Sjogren's syndrome. Joint Bone Spine 2018, 85(3):379-380.

12. Flach C-F, Qadri F, Bhuiyan TR, Alam NH, Jennische E, Lönnroth I, Holmgren J: Broad Up-Regulation of Innate Defense Factors during Acute Cholera. 2007, 75(5):2343-2350.

13. Zlotnik A, Yoshie O: The chemokine superfamily revisited. Immunity 2012, 36(5):705-716.

14. Choreno-Parra JA, Thirunavukkarasu S, Zuniga J, Khader SA: The protective and pathogenic roles of CXCL17 in human health and disease: Potential in respiratory medicine. Cytokine Growth Factor Rev 2020, 53:53-62.

15. Ardain A, Domingo-Gonzalez R, Das S, Kazer SW, Howard NC, Singh A, Ahmed M, Nhamoyebonde S, Rangel-Moreno J, Ogongo P et al: Group 3 innate lymphoid cells mediate early protective immunity against tuberculosis. Nature 2019, 570(7762):528-532.

16. Choreno-Parra JA, Dunlap M, Swanson R, Jiménez-Álvarez LA, Zúñiga J, Khader SA: The C-X-C motif chemokine 17 plays a minimal role during lung infection with hypervirulent Mycobacterium tuberculosis. 2020, 204(1 Supplement):85.13-85.13.

17. Zhou Z, Ren L, Zhang L, Zhong J, Xiao Y, Jia Z, Guo L, Yang J, Wang C, Jiang S et al: Heightened Innate Immune Responses in the Respiratory Tract of COVID-19 Patients. Cell host \& microbe 2020, 27(6):883-890.e882.

18. Cao Y, Huang Y, Xu K, Liu Y, Li X, Xu Y, Zhong W, Hao P: Differential responses of innate immunity triggered by different subtypes of influenza a viruses in human and avian hosts. BMC Med Genomics 2017, 10(Suppl 4):70.

19. Influenza Research Database (IRD). Available from: https://www.fludb.org/brc/hostFactorExperimentDetails.spg? method=SubmitForm\&fromDetail=true\&biosetlds=1320\&expSeqld=178\&resultMatrixUserDefld=SCL008_R_N_RM\&decorator=influenza\# (Accessed July 7th 2020) [https://www.fludb.org/brc/hostFactorExperimentDetails.spg?

method=SubmitForm\&fromDetail=true\&biosetlds=1320\&expSeqld=178\&resultMatrixUserDefld=SCL008_R_N_RM\&decorator=influenza\# ]

20. Lee WY, Wang CJ, Lin TY, Hsiao CL, Luo CW: CXCL17, an orphan chemokine, acts as a novel angiogenic and anti-inflammatory factor. Am J Physiol Endocrinol Metab 2013, 304(1):E32-40.

21. Tang X, Du R-H, Wang R, Cao T-Z, Guan L-L, Yang C-Q, Zhu Q, Hu M, Li X-Y, Li Y et al: Comparison of Hospitalized Patients With ARDS Caused by COVID-19 and H1N1. Chest 2020:S0012-3692(0020)30558-30554.

Page 10/29 
22. Jiang C, Yao X, Zhao Y, Wu J, Huang P, Pan C, Liu S, Pan C: Comparative review of respiratory diseases caused by coronaviruses and influenza A viruses during epidemic season. Microbes and infection 2020, 22(6-7):236-244.

23. Choreno-Parra JA, Jimenez-Alvarez LA, Cruz Lagunas A, Rodriguez-Reyna TS, Ramirez-Martinez G, Sandoval-Vega M, Hernandez-Garcia DL, Choreno-Parra EM, Balderas-Martinez YI, Martinez-Sanchez ME et al: Clinical and immunological factors that distinguish CoVID-19 from pandemic influenza A(H1N1). medRxiv 2020:2020.2008.2010.20170761.

24. Coates BM, Staricha KL, Koch CM, Cheng Y, Shumaker DK, Budinger GRS, Perlman H, Misharin AV, Ridge KM: Inflammatory Monocytes Drive Influenza A Virus-Mediated Lung Injury in Juvenile Mice. J Immunol 2018, 200(7):2391-2404.

25. Lin KL, Suzuki Y, Nakano H, Ramsburg E, Gunn MD: CCR2+ monocyte-derived dendritic cells and exudate macrophages produce influenza-induced pulmonary immune pathology and mortality. J Immuno/ 2008, 180(4):2562-2572.

26. Oka T, Sugaya M, Takahashi N, Takahashi T, Shibata S, Miyagaki T, Asano Y, Sato S: CXCL17 Attenuates Imiquimod-Induced Psoriasislike Skin Inflammation by Recruiting Myeloid-Derived Suppressor Cells and Regulatory T Cells. J Immuno/ 2017, 198(10):3897-3908.

27. Hernandez-Ruiz M, Othy S, Herrera C, Nguyen HT, Arrevillaga-Boni G, Catalan-Dibene J, Cahalan MD, Zlotnik A: Cxcl17(-/-) mice develop exacerbated disease in a T cell-dependent autoimmune model. J Leukoc Biol 2019, 105(5):1027-1039.

28. Wang W, Su B, Pang L, Qiao L, Feng Y, Ouyang Y, Guo X, Shi H, Wei F, Su X et al: High-dimensional immune profiling by mass cytometry revealed immunosuppression and dysfunction of immunity in COVID-19 patients. Cell Mol Immuno/ 2020, 17(6):650-652.

\section{Tables}




\begin{tabular}{|c|c|c|c|}
\hline \multirow[t]{2}{*}{ Characteristic } & Influenza & COVID-19 & $p$-value \\
\hline & $N=68$ & $N=24$ & \\
\hline Age (years), median (range) & $48(20-77)$ & $50(28-73)$ & 0.6536 \\
\hline Males & $48(70.5)$ & $18(75)$ & 0.7953 \\
\hline BMI & $33.4(30-38.2)$ & $28.9(25.2-30.3)$ & $<0.0001$ \\
\hline Comorbidities & 27 (39.7) & $5(20.8)$ & 0.1349 \\
\hline Smoking & $14(20.5)$ & $7(29.1)$ & 0.4063 \\
\hline Obesity & $18(26.4)$ & $5(20.8)$ & 0.7848 \\
\hline Diabetes & $4(5.8)$ & $0(0)$ & 0.5695 \\
\hline SAH & $3(4.4)$ & $1(4.1)$ & $>0.9999$ \\
\hline \multicolumn{4}{|l|}{ OSA } \\
\hline \multicolumn{4}{|l|}{ COPD } \\
\hline Symptoms at onset & $62(91.1)$ & $18(75)$ & 0.0725 \\
\hline Fever & $56(82.3)$ & $18(75)$ & 0.5500 \\
\hline Myalgia & $53(79.1)$ & $16(66.6)$ & 0.2847 \\
\hline Arthralgia & $33(48.52)$ & $11(45.8)$ & $>0.9999$ \\
\hline Headache & $65(95.5)$ & $16(66.6)$ & 0.0007 \\
\hline Dyspnea & $13(19.1)$ & $1(4.1)$ & 0.1038 \\
\hline Nasal congestion & $26(38.2)$ & $3(12.5)$ & 0.0221 \\
\hline Rhinorrhea & $24(35.8)$ & $4(16.6)$ & 0.1223 \\
\hline Sore throat & $9(13.2)$ & $0(0)$ & 0.1053 \\
\hline Thoracic pain & $66(97)$ & $21(87.5)$ & 0.1093 \\
\hline Cough & $38(55.8)$ & $2(8.3)$ & $<0.0001$ \\
\hline Sputum & $27(40.2)$ & $19(79.1)$ & 0.0017 \\
\hline Dry cough & $49(72)$ & $18(75)$ & $>0.9999$ \\
\hline Fatigue & $5(7.35)$ & $5(20.8)$ & 0.1196 \\
\hline Diarrhea & $4(5.88)$ & $3(12.5)$ & 0.3717 \\
\hline Nausea & $2(2.9)$ & $4(16.6)$ & 0.0382 \\
\hline \multicolumn{4}{|l|}{ Vomit } \\
\hline \multicolumn{3}{|l|}{ (days) } & 0.3756 \\
\hline Vital signs at admission & $38(37-38)$ & 37 (37-37.08) & 0.0018 \\
\hline Body temperature $\left({ }^{\circ} \mathrm{C}\right)$ & $25(20-30)$ & $24(22-26)$ & 0.3486 \\
\hline Respiratory rate (bpm) & $96(85-108)$ & $85(75-96)$ & 0.0074 \\
\hline Hearth rate (bpm) & $85.4(75-118.3)$ & $77.5(71-87)$ & 0.0677 \\
\hline \multicolumn{4}{|l|}{ MAP $(\mathrm{mmHg})$} \\
\hline Glucose (mg/dL) & 144.9 (114.9-221.4) & 115.6 (96.7-200.7) & 0.0771 \\
\hline \multirow[t]{2}{*}{ Blood count } & $7.2(5.6-10.3)$ & $9.1(5.2-12.2)$ & 0.4429 \\
\hline & $5.7(4.5-8.3)$ & $7.4(3.6-10.1)$ & 0.7057 \\
\hline
\end{tabular}

Page 12/29 
White blood cells $\left(10^{9} / \mathrm{L}\right)$
$0.8(0.5-1.1)$

$8.5(5.4-12.6)$

14.9 (13.3-17.3)

$177.5(136.5-216.5)$
$0.8(0.6-1.0)$

0.4657

Neutrophils $\left(10^{9} / \mathrm{L}\right)$

Lymphocytes $\left(10^{9} / \mathrm{L}\right)$

NLR

$\mathrm{Hgb}(\mathrm{g} / \mathrm{dL})$

Platelets $\left(10^{9} / \mathrm{L}\right)$

\section{Renal function}

$\mathrm{Cr}(\mathrm{mg} / \mathrm{dL})$

BUN (mg/dL)

$\mathrm{Na}(\mathrm{mmol} / \mathrm{L})$

$\mathrm{K}(\mathrm{mmol} / \mathrm{L})$

\section{Liver function}

Total bilirubin ( $\mathrm{mg} / \mathrm{dL})$

AST (U/L)

ALT (U/L)

\section{Other biomarkers}

LDH (U/L)

$\operatorname{ALP}(U / L)$

CPK (U/L)

Procalcitonin $(\mathrm{ng} / \mathrm{mL})$

\section{$\mathrm{PaO}_{2} / \mathrm{FiO}_{2}$}

Severity of illness scores

SOFA

APACHE II

\section{Respiratory support}

High flow nasal cannula

MV

Prone position

ECMO

$$
1.02 \text { (0.7-1.4) }
$$

24.9 (15.5-35.7

$137.4(133.4-140.5)$

$4.1(3.8-4.5)$
$8.7(3.9-13.4)$

14.1 (13.2-15.3)

0.1015

$202(145.8-256.8)$
0.7927

$1.0(0.8-1.4)$

$$
18.3 \text { (13.6-26.8) }
$$

$138.6(136-141.5)$

4.1 (3.9-4.3)
0.8754

0.1390

0.2155

0.9384
$0.6(0.4-0.8)$

$61.8(44.7-87)$

41 (26.3-52.2)
$0.4(0.3-0.7)$

35.1 (23.6-80.7)

32.7 (25.1-48.4)
0.0900

0.0055

0.2768
643.8 (500-877.9)

121.7 (98.2-161.1)

274.4 (108-700.8)

0.55 (0.15-1.92)
341.7 (251.5-477.7)

78 (67.7-88.2)

117.8 (64-117.8)

$0.1(0.05-0.17)$
$<0.0001$

$<0.0001$

0.4754

$<0.0001$
82.5 (59.9-143.5)

7 (5-9)

10 (7-16)
127.8 (94.7-198.1)

$5(3-6)$

7 (4-8)
0.0031

0.0002

0.0069
0 (0)

$68(100)$

40 (58.8)

$7(10.2)$
7 (29.1)

$17(70.8)$

$8(33.3)$

$0(0)$
$<0.0001$

$<0.0001$

0.0359

0.1836

\begin{tabular}{llll} 
Renal replacement therapy & $16(23.5)$ & $2(8.3)$ & 0.1399 \\
\hline Mortality & $16(23.5)$ & $10(41.6)$ & 0.1151
\end{tabular}

Data are displayed as $\mathrm{n}(\%)$ or median (IQR). N is the total number of patients with available data. ALP, alkaline phosphatase; APACHE-II, Acute Physiology And Chronic Health Evaluation II; AST, aspartate aminotransferase; ALT, alanine aminotransferase; BMI, body mass index; bpm, breaths/beats per minute; BUN, blood ureic nitrogen; COPD, chronic obstructive pulmonary disease; CPK, creatine phosphokinase; Cr, creatinine; ECMO, extra-corporeal membrane oxygenation; FiO2, fraction of inspired oxygen; HCO3, bicarbonate; Hgb, hemoglobin; IQR, interquartile range; ICU, intensive care unit; LDH, lactate dehydrogenase; MAP, mean arterial pressure; MV, mechanical ventilation; ND, not determined; NLR, neutrophil/lymphocyte ration; OSA, obstructive sleep apnea syndrome; PaO2, partial pressure of oxygen in arterial blood; PCO2, partial pressure of carbon dioxide in blood; SAH, systemic arterial hypertension; SD, standard deviation; SOFA, Sequential Organ Failure Assessment. Differences in continuous variables were estimated using the Mann Whitney U test. Differences in categorical variables were calculated using the Fisher's exact or the Chi square test as appropriate. 


\begin{tabular}{|c|c|c|}
\hline \multirow[t]{2}{*}{ Time after hospital admission } & \multicolumn{2}{|l|}{ Survival $(\%, 95 \% \mathrm{Cl})$} \\
\hline & $\mathrm{CXCL} 17<1,128 \mathrm{pg} / \mathrm{mL}$ & $\mathrm{CXCL} 17 \geq 1,128 \mathrm{pg} / \mathrm{mL}$ \\
\hline 7 days & 96.66 (78.6-99.52) & $94.73(80.55-98.65)$ \\
\hline 14 days & $84.86(64.38-94.06)$ & $91.776(76.56-97.27)$ \\
\hline 21 days & $84.86(64.38-94.06)$ & $85.06(67.5-94.16)$ \\
\hline 28 days & $84.86(64.38-94.06)$ & $63.33(41.48-78.89)$ \\
\hline 60 days & $84.86(64.38-94.06)$ & $31.66(1.93-71.53)$ \\
\hline
\end{tabular}

\section{Figures}

a

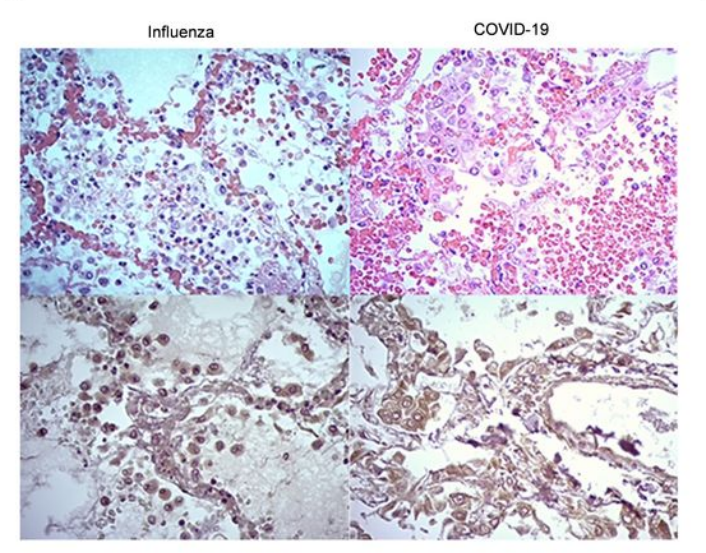

c

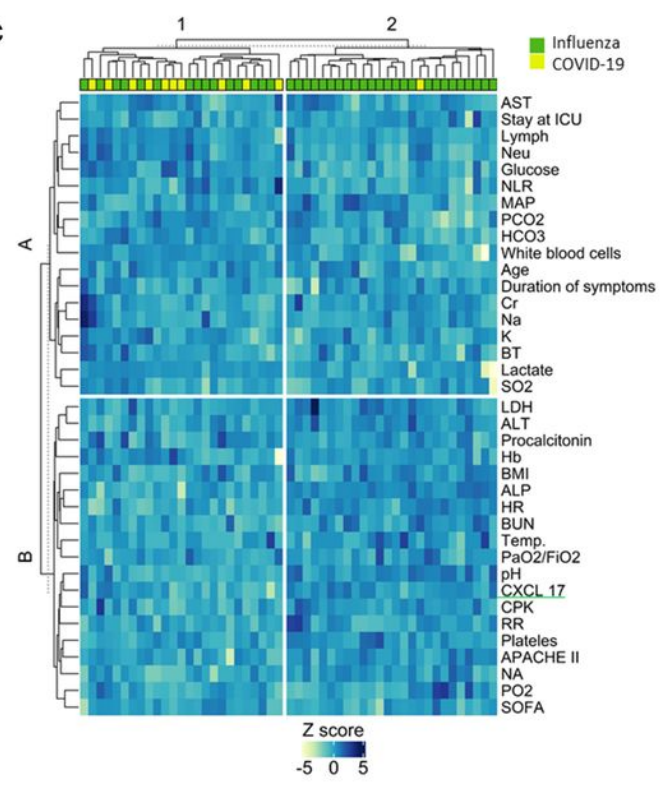

b

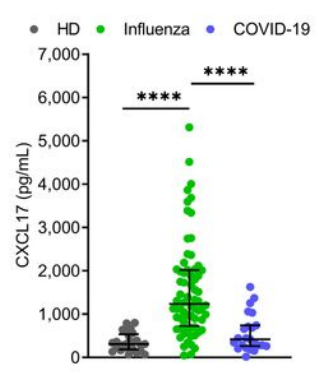

e

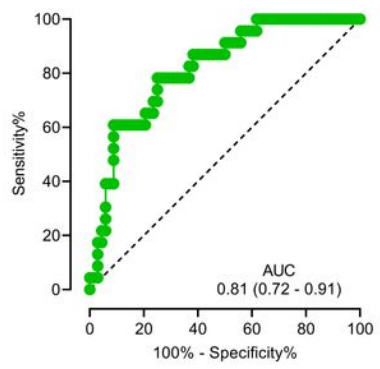

d

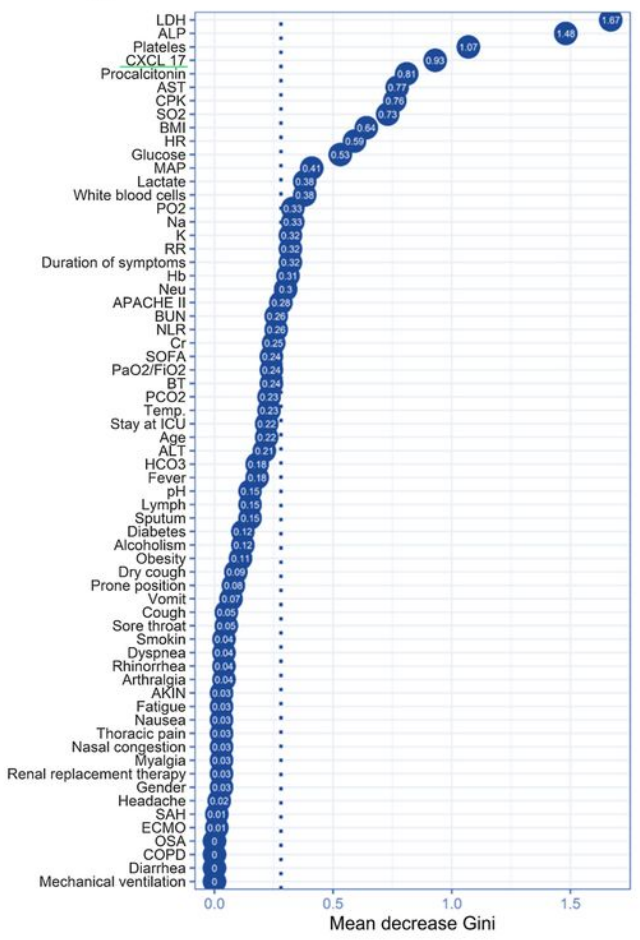

Figure 1

CXCL17 in the lung and serum of influenza and COVID-19 patients. (a) Lung autopsy specimens were obtained from influenza and COVID-19 patients. Left upper panel: Influenza-induced lung inflammatory infiltrate occupying alveolar spaces and composed of macrophages, polymorphonuclear cells, fibrin deposits, and hemorrhage. H\&E, X400. Right upper panel: SARS-CoV-2 induced extensive lung inflammation, 
thickening of the alveolar walls, partial loss of the histological architecture, and hemorrhage. The inflammatory infiltrate was mainly composed of macrophages. H\&E, X400. Left lower panel: Influenza triggers the expression of CXCL17 in macrophages and alveolar epithelial cells. IHQ, X400. Right lower panel: SARS-CoV-2-induced expression of CXCL17 in macrophages and alveolar epithelial cells. IHQ, X400. (b) Serum CXCL17 levels in healthy donors (HD; $n=30)$ patients with influenza $(n=68)$, and COVID-19 subjects $(n=24)$. Kruskal-Wallis test and post hoc Dunn test (plots display medians with interquartile ranges; ${ }^{* \star *} \mathrm{p} \leq 0.0001$ ). (c) $\mathrm{K}$-means clustering analysis of the clinical characteristics of influenza and COVID-19 patients. (d) Random forest algorithm showing the most important factors that differentiate influenza from COVID-19. The points represent the mean decrease Gini values, indicative of the importance of each variable with respect to the mean importance of the model (discontinuous vertical line). (e) Receiver operating characteristic (ROC) curve of the levels of CXCL17 in influenza and COVID-19 subjects. The graph displays area under the curve (AUC) and 95\% $\mathrm{Cl}$ interval values.

a

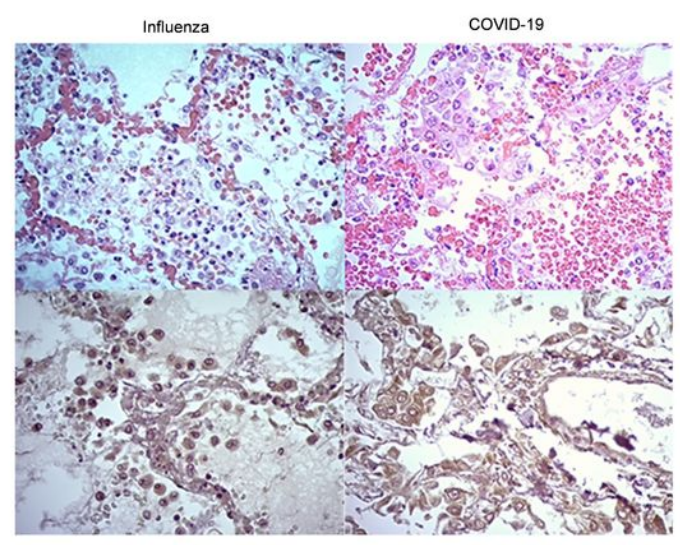

C

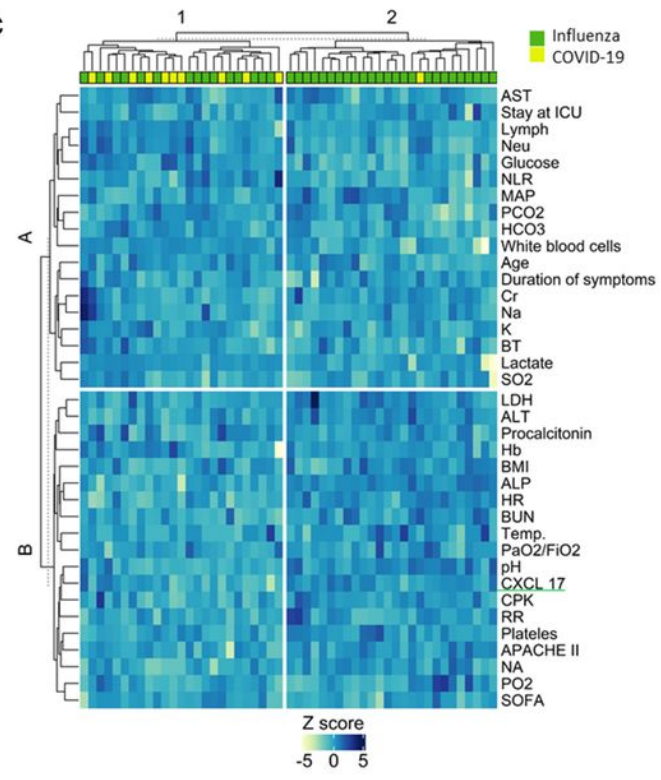

b

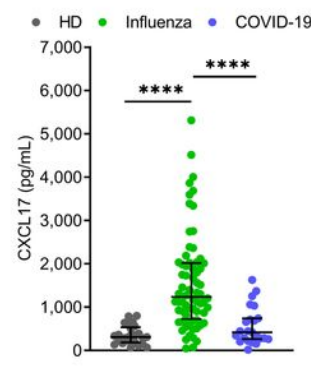

e

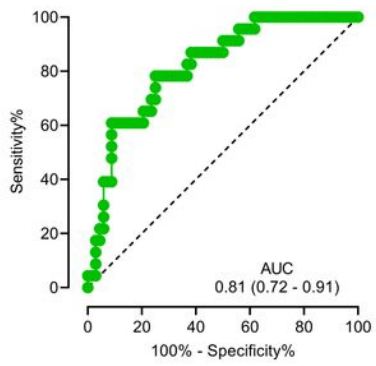

d

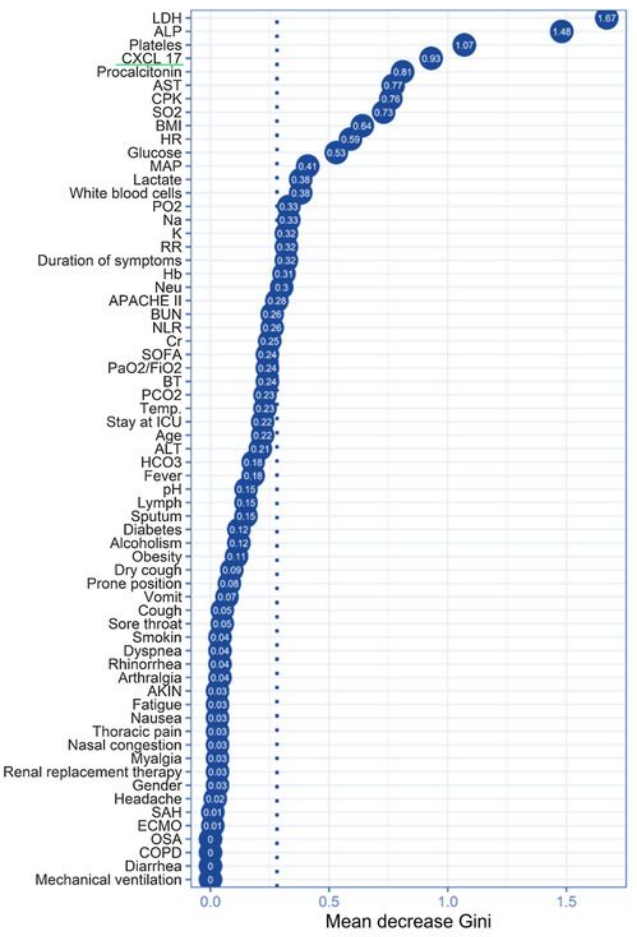

Figure 1

CXCL17 in the lung and serum of influenza and COVID-19 patients. (a) Lung autopsy specimens were obtained from influenza and COVID-19 patients. Left upper panel: Influenza-induced lung inflammatory infiltrate occupying alveolar spaces and composed of macrophages, polymorphonuclear cells, fibrin deposits, and hemorrhage. H\&E, X400. Right upper panel: SARS-CoV-2 induced extensive lung inflammation, thickening of the alveolar walls, partial loss of the histological architecture, and hemorrhage. The inflammatory infiltrate was mainly composed of macrophages. H\&E, X400. Left lower panel: Influenza triggers the expression of CXCL17 in macrophages and alveolar epithelial cells. IHQ, X400. Right lower panel: SARS-CoV-2-induced expression of CXCL17 in macrophages and alveolar epithelial cells. IHQ, X400. (b) Serum CXCL17 levels in healthy donors (HD; $n=30)$ patients with influenza $(n=68)$, and COVID-19 subjects $(n=24)$. Kruskal-Wallis test and post hoc Dunn test (plots display medians with interquartile ranges; $* \star \star * \leq 0.0001$ ). (c) K-means clustering analysis of the clinical characteristics of influenza and COVID-19 patients. (d) Random forest algorithm showing the most important factors that differentiate 
influenza from COVID-19. The points represent the mean decrease Gini values, indicative of the importance of each variable with respect to the mean importance of the model (discontinuous vertical line). (e) Receiver operating characteristic (ROC) curve of the levels of CXCL17 in influenza and COVID-19 subjects. The graph displays area under the curve (AUC) and $95 \% \mathrm{Cl}$ interval values.

a

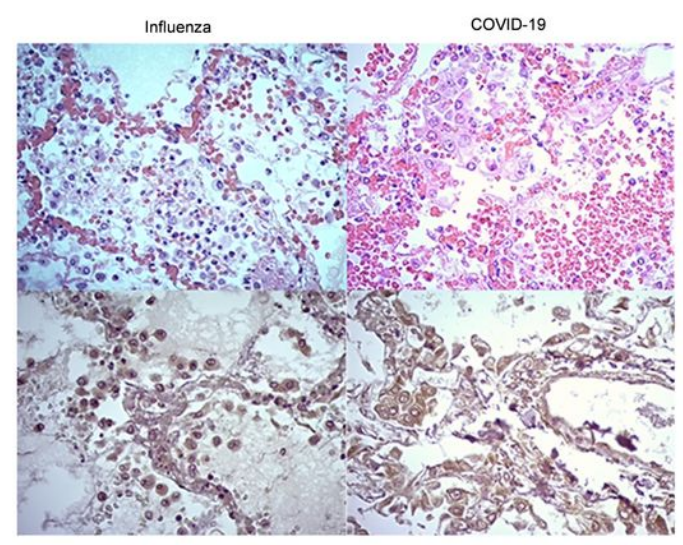

C

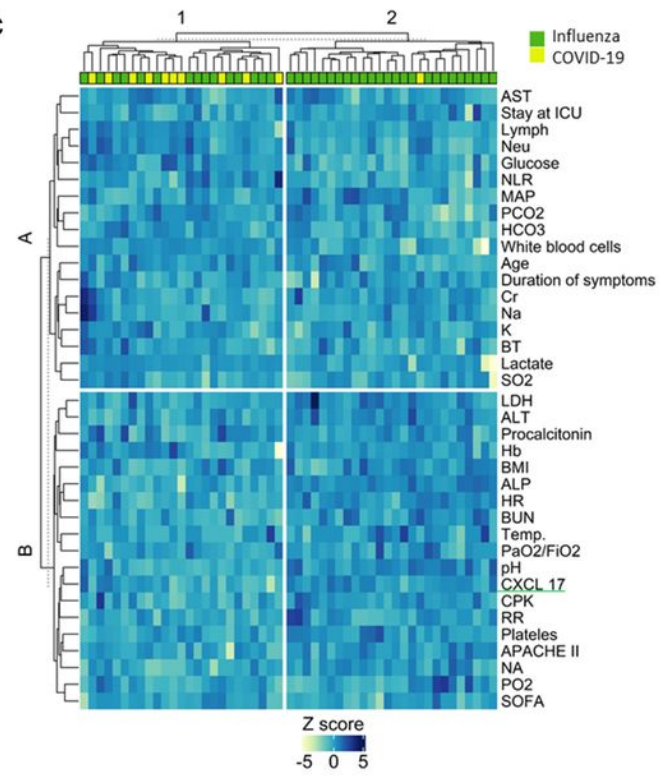

b

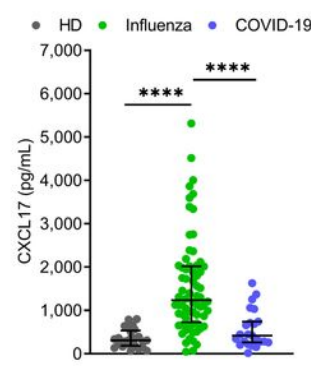

e

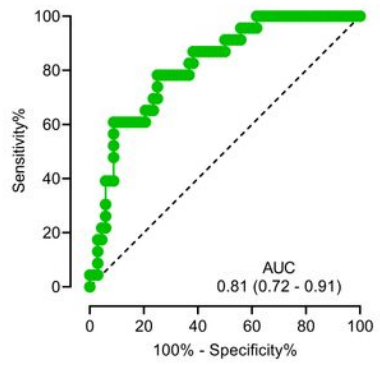

d

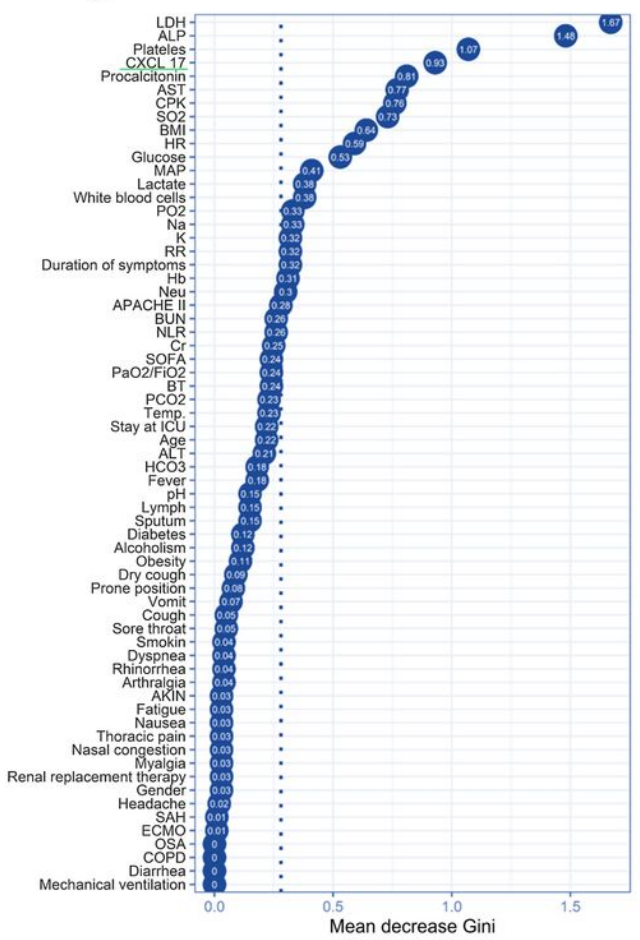

Figure 1

CXCL17 in the lung and serum of influenza and COVID-19 patients. (a) Lung autopsy specimens were obtained from influenza and COVID-19 patients. Left upper panel: Influenza-induced lung inflammatory infiltrate occupying alveolar spaces and composed of macrophages, polymorphonuclear cells, fibrin deposits, and hemorrhage. H\&E, X400. Right upper panel: SARS-CoV-2 induced extensive lung inflammation, thickening of the alveolar walls, partial loss of the histological architecture, and hemorrhage. The inflammatory infiltrate was mainly composed of macrophages. H\&E, X400. Left lower panel: Influenza triggers the expression of CXCL17 in macrophages and alveolar epithelial cells. IHQ, X400. Right lower panel: SARS-CoV-2-induced expression of CXCL17 in macrophages and alveolar epithelial cells. IHQ, X400. (b) Serum CXCL17 levels in healthy donors (HD; $n=30)$ patients with influenza ( $n=68)$, and COVID-19 subjects ( $n=24)$. Kruskal-Wallis test and post hoc Dunn test (plots display medians with interquartile ranges; $\left.{ }^{* \star *} \mathrm{p} \leq 0.0001\right)$. (c) K-means clustering analysis of the clinical characteristics of influenza and COVID-19 patients. (d) Random forest algorithm showing the most important factors that differentiate influenza from COVID-19. The points represent the mean decrease Gini values, indicative of the importance of each variable with respect to the mean importance of the model (discontinuous vertical line). (e) Receiver operating characteristic (ROC) curve of the levels of CXCL17 in influenza and COVID-19 subjects. The graph displays area under the curve (AUC) and 95\% $\mathrm{Cl}$ interval values. 
a

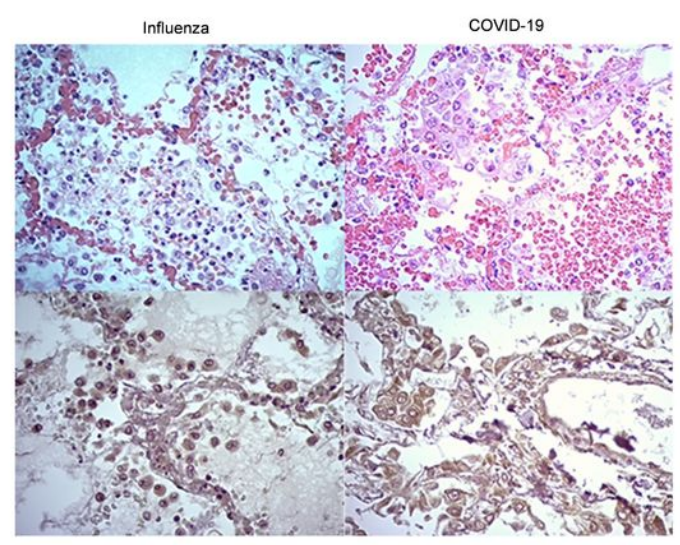

C

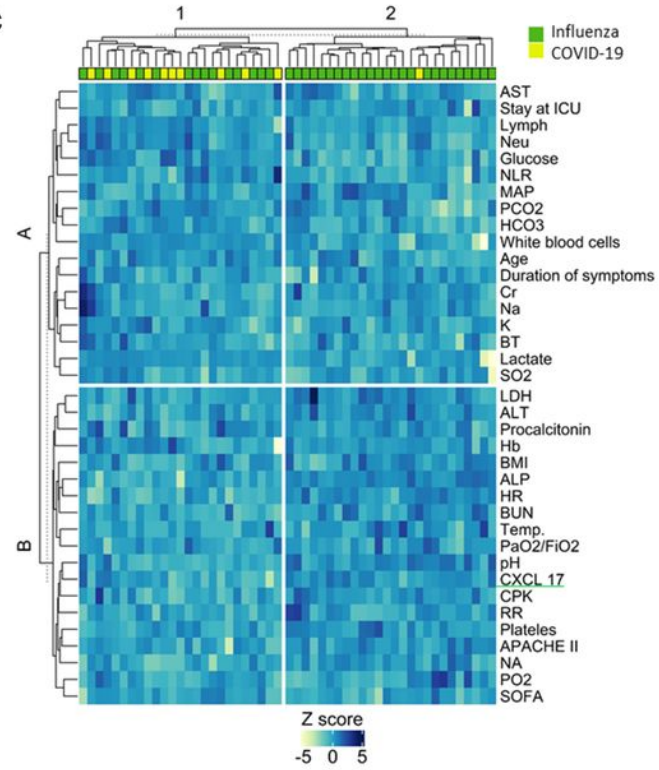

b

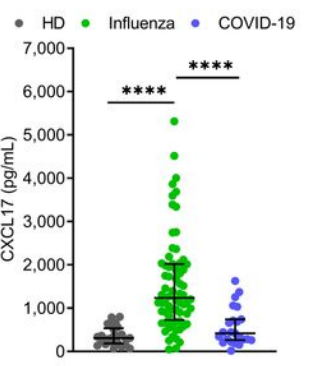

e

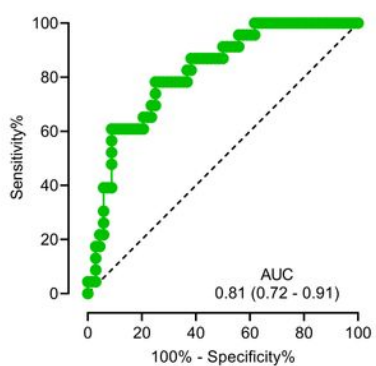

d

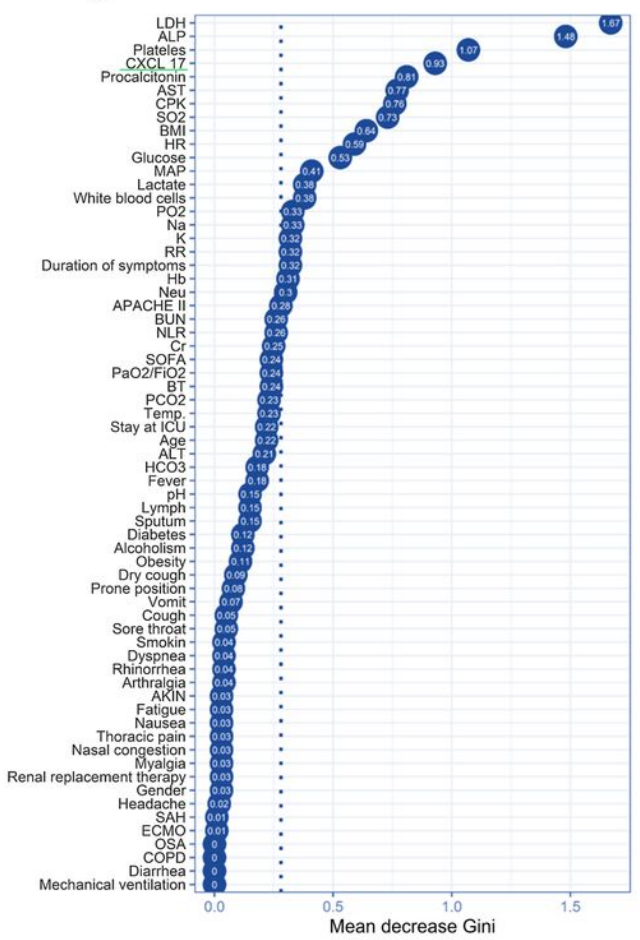

Figure 1

CXCL17 in the lung and serum of influenza and COVID-19 patients. (a) Lung autopsy specimens were obtained from influenza and COVID-19 patients. Left upper panel: Influenza-induced lung inflammatory infiltrate occupying alveolar spaces and composed of macrophages, polymorphonuclear cells, fibrin deposits, and hemorrhage. H\&E, X400. Right upper panel: SARS-CoV-2 induced extensive lung inflammation, thickening of the alveolar walls, partial loss of the histological architecture, and hemorrhage. The inflammatory infiltrate was mainly composed of macrophages. H\&E, X400. Left lower panel: Influenza triggers the expression of CXCL17 in macrophages and alveolar epithelial cells. IHQ, X400. Right lower panel: SARS-CoV-2-induced expression of CXCL17 in macrophages and alveolar epithelial cells. IHQ, X400. (b) Serum CXCL17 levels in healthy donors (HD; $n=30)$ patients with influenza ( $n=68)$, and COVID-19 subjects ( $n=24)$. Kruskal-Wallis

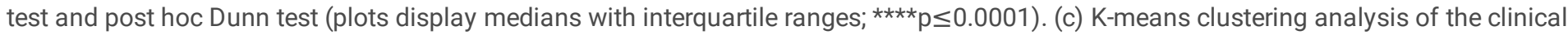
characteristics of influenza and COVID-19 patients. (d) Random forest algorithm showing the most important factors that differentiate influenza from COVID-19. The points represent the mean decrease Gini values, indicative of the importance of each variable with respect to the mean importance of the model (discontinuous vertical line). (e) Receiver operating characteristic (ROC) curve of the levels of CXCL17 in influenza and COVID-19 subjects. The graph displays area under the curve (AUC) and 95\% $\mathrm{Cl}$ interval values. 
a

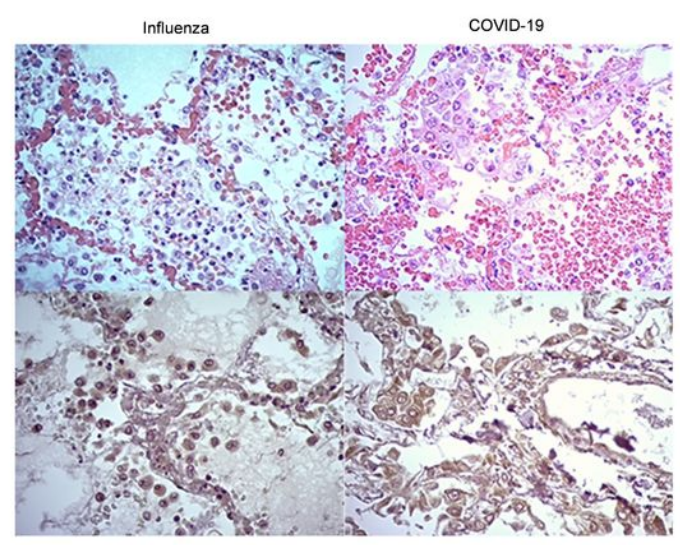

C

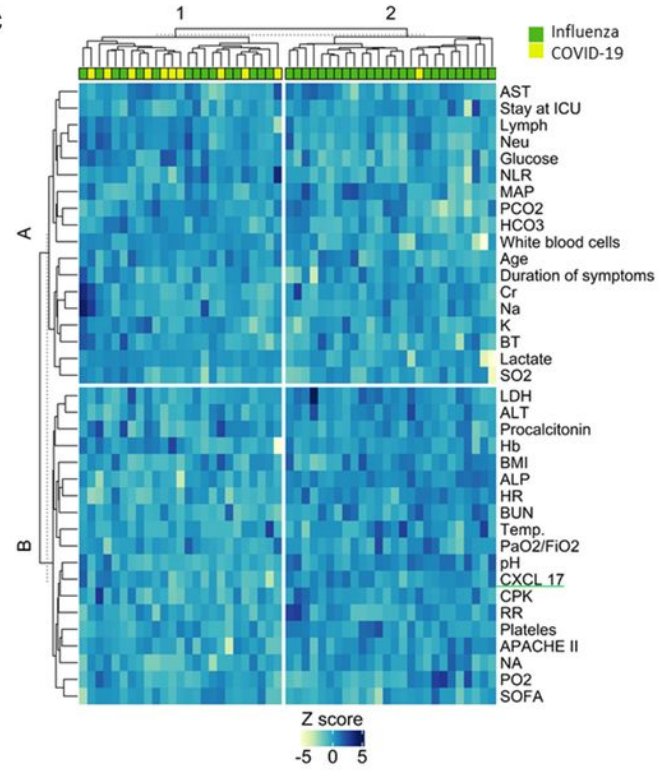

b

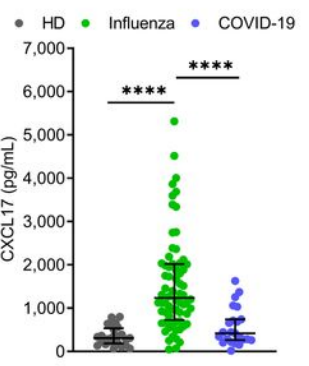

e

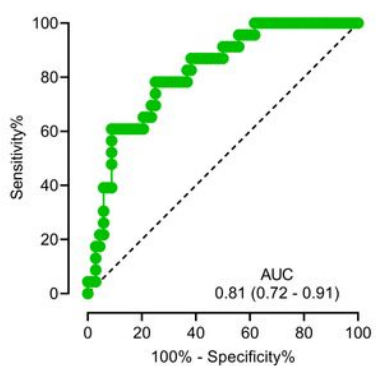

d

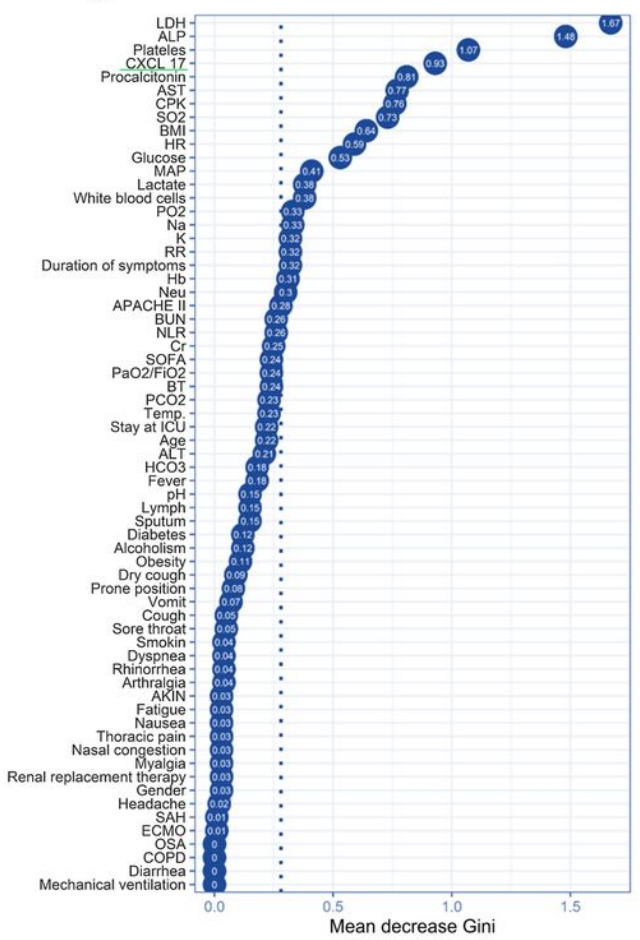

Figure 1

CXCL17 in the lung and serum of influenza and COVID-19 patients. (a) Lung autopsy specimens were obtained from influenza and COVID-19 patients. Left upper panel: Influenza-induced lung inflammatory infiltrate occupying alveolar spaces and composed of macrophages, polymorphonuclear cells, fibrin deposits, and hemorrhage. H\&E, X400. Right upper panel: SARS-CoV-2 induced extensive lung inflammation, thickening of the alveolar walls, partial loss of the histological architecture, and hemorrhage. The inflammatory infiltrate was mainly composed of macrophages. H\&E, X400. Left lower panel: Influenza triggers the expression of CXCL17 in macrophages and alveolar epithelial cells. IHQ, X400. Right lower panel: SARS-CoV-2-induced expression of CXCL17 in macrophages and alveolar epithelial cells. IHQ, X400. (b) Serum CXCL17 levels in healthy donors (HD; $n=30)$ patients with influenza ( $n=68)$, and COVID-19 subjects ( $n=24)$. Kruskal-Wallis

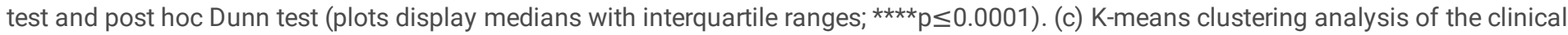
characteristics of influenza and COVID-19 patients. (d) Random forest algorithm showing the most important factors that differentiate influenza from COVID-19. The points represent the mean decrease Gini values, indicative of the importance of each variable with respect to the mean importance of the model (discontinuous vertical line). (e) Receiver operating characteristic (ROC) curve of the levels of CXCL17 in influenza and COVID-19 subjects. The graph displays area under the curve (AUC) and 95\% $\mathrm{Cl}$ interval values. 
a

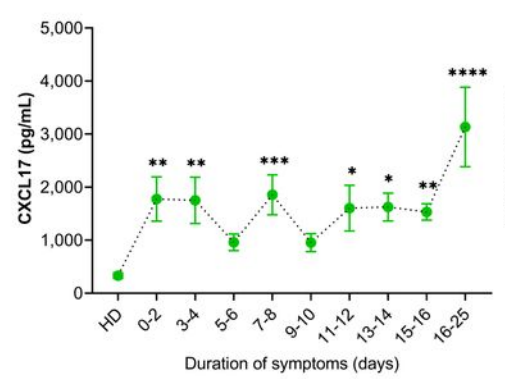

Duration of symptoms (days)

e

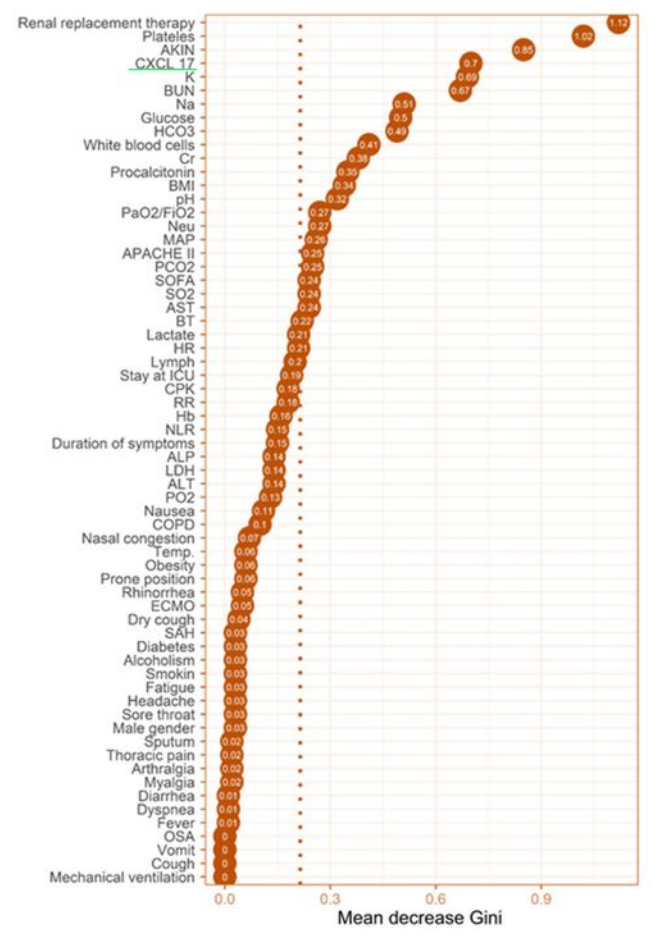

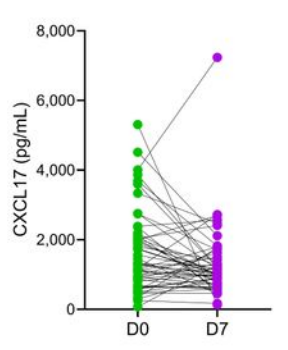

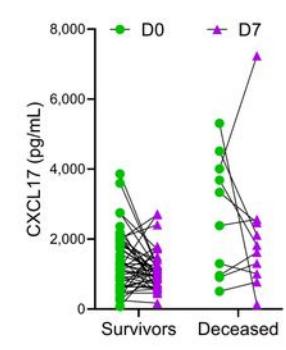

d
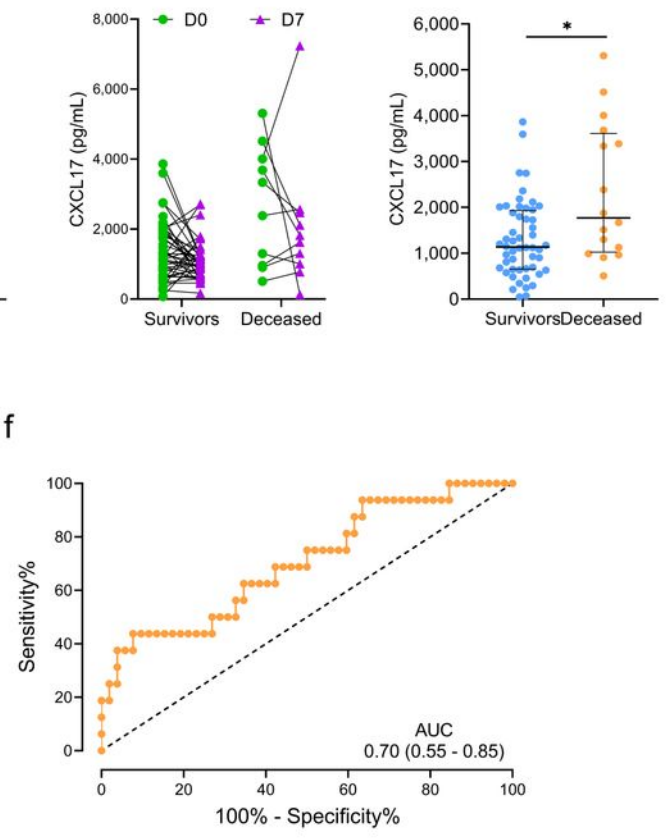

g

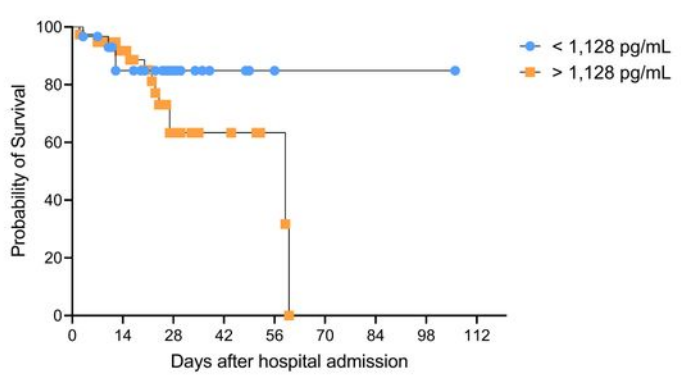

Figure 2

Dynamics and prognostic value of serum CXCL17 levels in influenza (a) Influenza patients were grouped according to their duration of symptoms on admission. Levels of CXCL17 were compared to healthy donors (HD; Kruskal-Wallis test and post hoc Dunn test; graph displays mean CXCL17 levels and the standard error $\left.( \pm S E) ;{ }^{*} \leq \leq 0.05, * * p \leq 0.01, * * * p \leq 0.001, * * * * p \leq 0.0001\right)$. (b) We obtained a second serum sample from influenza subjects seven days (D7) after hospital admission (D0). Levels of CXCL17 at D0 and D7 were compared with the Mann-Whitney U test. (c) Same comparison was performed in survivors and deceased patients (Wilcoxon test in each group). (d) Serum CXCL17 levels in survivor and deceased influenza patients (Mann-Whitney U test; plots display medians and interquartile ranges). (e) Random forest algorithm showing the most important factors that impact on influenza-assocaited mortality. The points represent the mean decrease Gini values, indicative of the importance of each variable with respect to the mean importance of the model (discontinuous vertical line). (f) Receiver operating characteristic (ROC) curve of the levels of CXCL17 in survivor and deceased influenza subjects. The graph displays area under the curve (AUC) and 95\% Cl interval values. (g) Survival curves of influenza patients grouped according to their serum CXCL17 levels were compared with the log-rank test. 
a

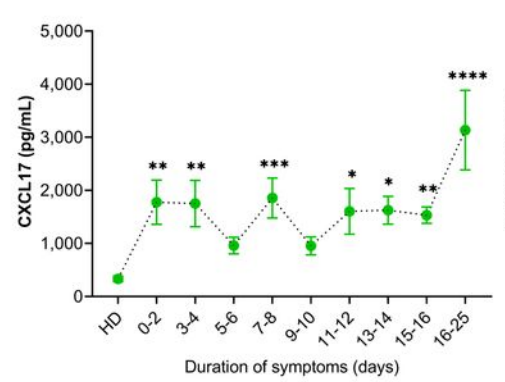

b

e

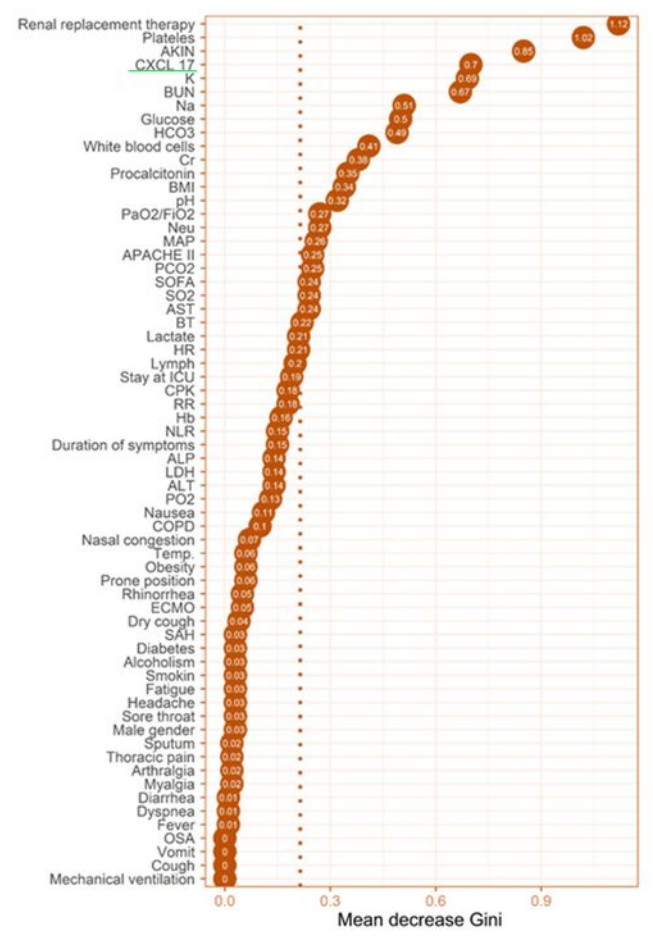

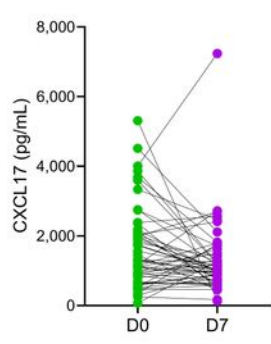
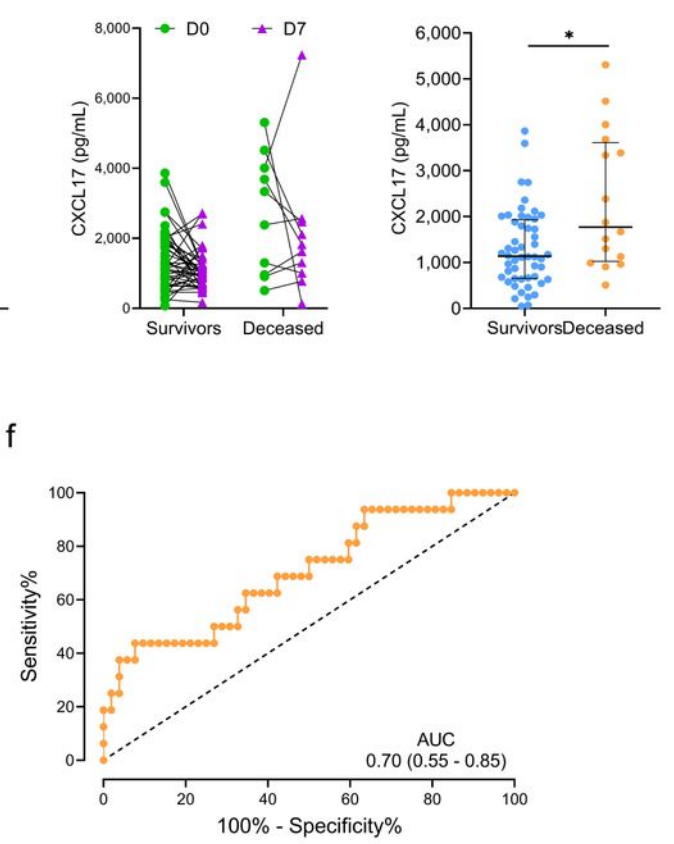

g

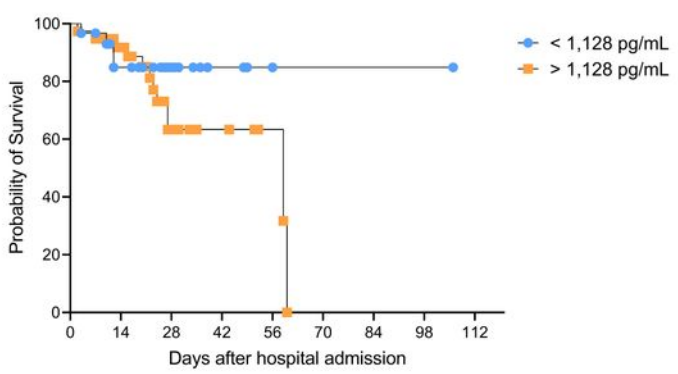

Figure 2

Dynamics and prognostic value of serum CXCL17 levels in influenza (a) Influenza patients were grouped according to their duration of symptoms on admission. Levels of CXCL17 were compared to healthy donors (HD; Kruskal-Wallis test and post hoc Dunn test; graph displays mean CXCL17 levels and the standard error ( \pm SE); $\left.{ }^{\star} p \leq 0.05, * \star p \leq 0.01,{ }^{* \star *} \mathrm{p} \leq 0.001,{ }^{* \star * *} \mathrm{p} \leq 0.0001\right)$. (b) We obtained a second serum sample from influenza subjects seven days (D7) after hospital admission (D0). Levels of CXCL17 at D0 and D7 were compared with the Mann-Whitney U test. (c) Same comparison was performed in survivors and deceased patients (Wilcoxon test in each group). (d) Serum CXCL17 levels in survivor and deceased influenza patients (Mann-Whitney U test; plots display medians and interquartile ranges). (e) Random forest algorithm showing the most important factors that impact on influenza-assocaited mortality. The points represent the mean decrease Gini values, indicative of the importance of each variable with respect to the mean importance of the model (discontinuous vertical line). (f) Receiver operating characteristic (ROC) curve of the levels of CXCL17 in survivor and deceased influenza subjects. The graph displays area under the curve (AUC) and 95\% Cl interval values. (g) Survival curves of influenza patients grouped according to their serum CXCL17 levels were compared with the log-rank test. 
a

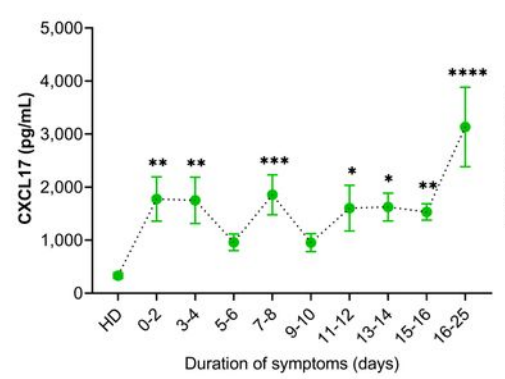

Duration of symptoms (days)

e

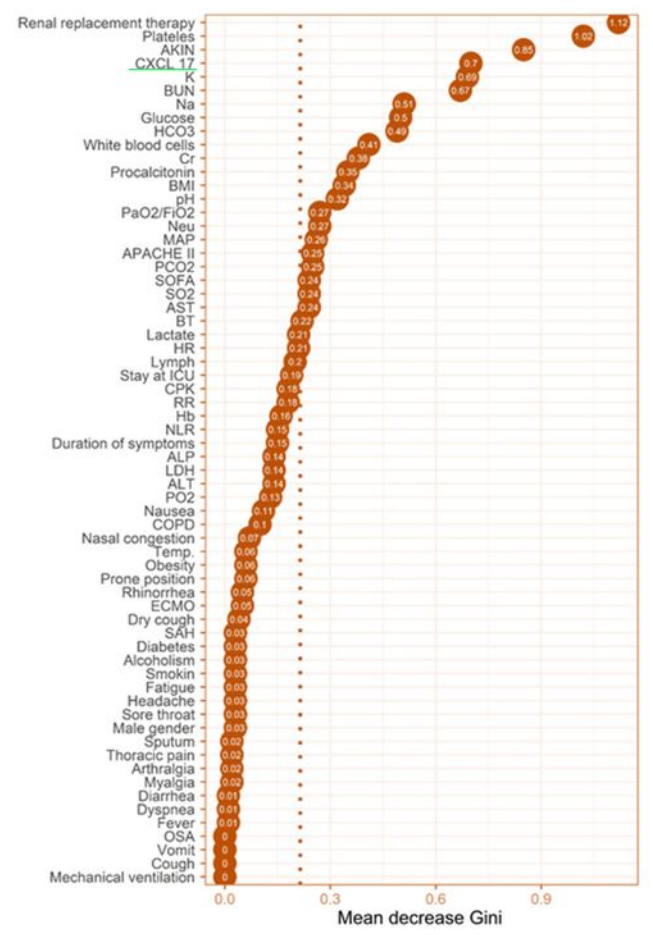

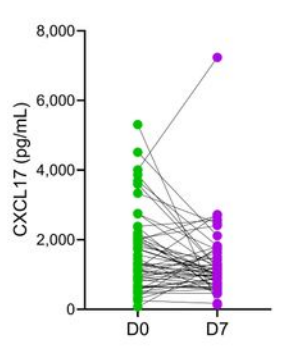

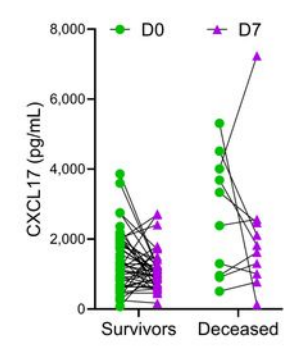

d
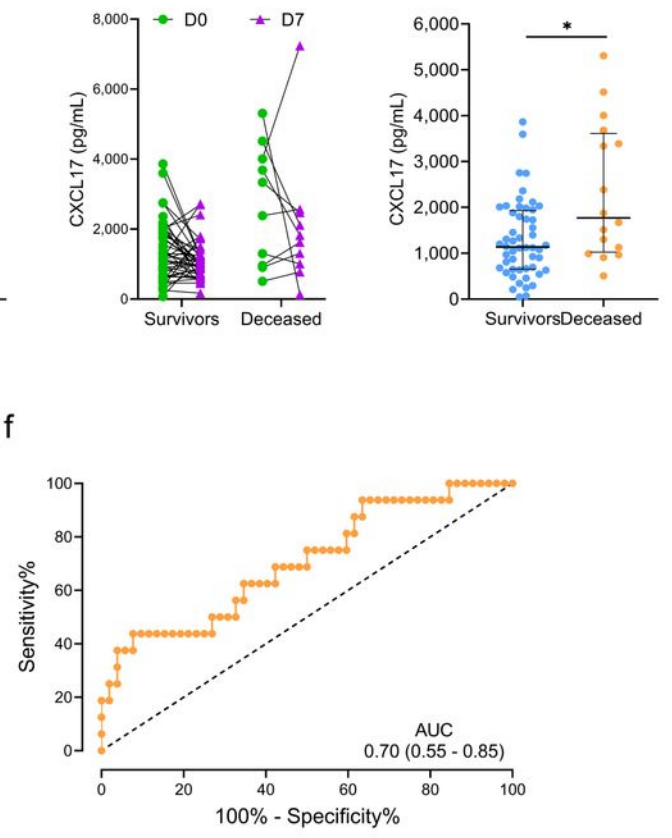

g

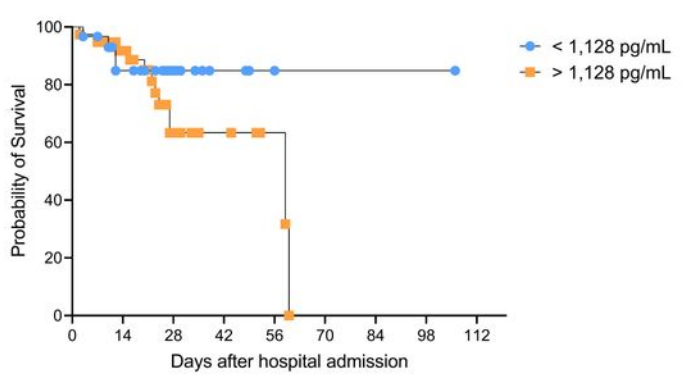

Figure 2

Dynamics and prognostic value of serum CXCL17 levels in influenza (a) Influenza patients were grouped according to their duration of symptoms on admission. Levels of CXCL17 were compared to healthy donors (HD; Kruskal-Wallis test and post hoc Dunn test; graph displays mean CXCL17 levels and the standard error $\left.( \pm S E) ;{ }^{*} \leq \leq 0.05, * * p \leq 0.01, * * * p \leq 0.001, * * * * p \leq 0.0001\right)$. (b) We obtained a second serum sample from influenza subjects seven days (D7) after hospital admission (D0). Levels of CXCL17 at D0 and D7 were compared with the Mann-Whitney U test. (c) Same comparison was performed in survivors and deceased patients (Wilcoxon test in each group). (d) Serum CXCL17 levels in survivor and deceased influenza patients (Mann-Whitney U test; plots display medians and interquartile ranges). (e) Random forest algorithm showing the most important factors that impact on influenza-assocaited mortality. The points represent the mean decrease Gini values, indicative of the importance of each variable with respect to the mean importance of the model (discontinuous vertical line). (f) Receiver operating characteristic (ROC) curve of the levels of CXCL17 in survivor and deceased influenza subjects. The graph displays area under the curve (AUC) and 95\% Cl interval values. (g) Survival curves of influenza patients grouped according to their serum CXCL17 levels were compared with the log-rank test. 
a

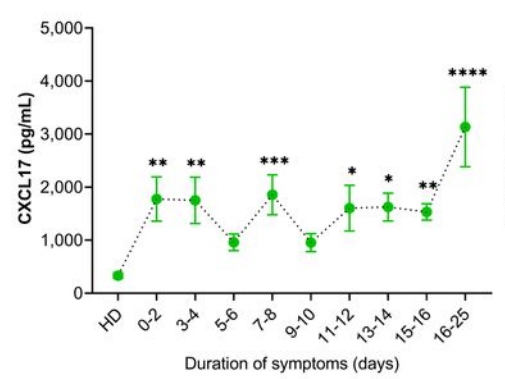

Duration of symptoms (days)

e

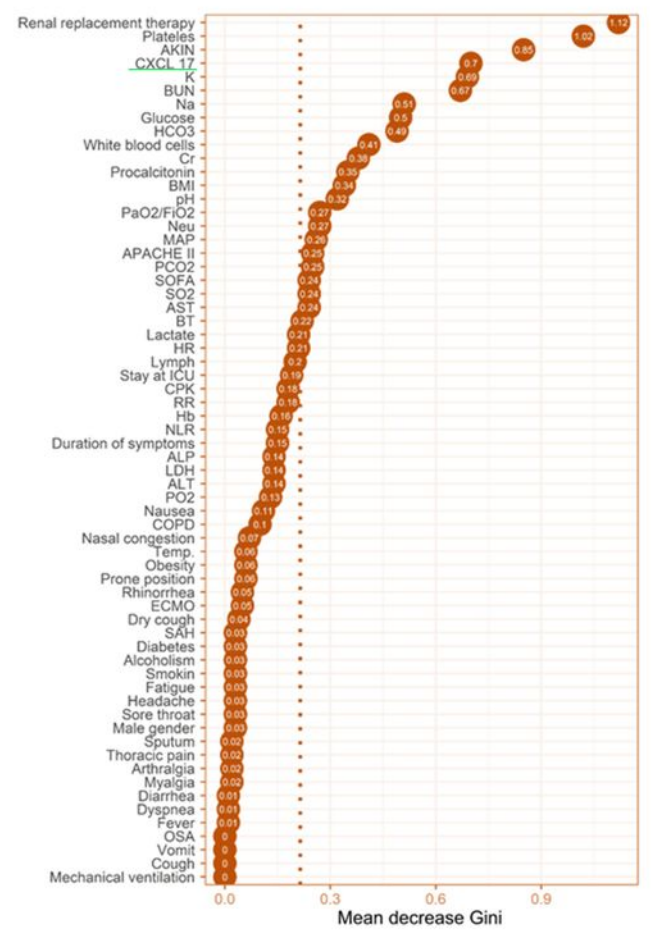

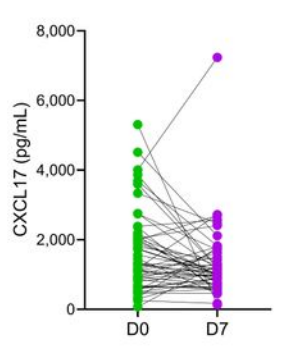

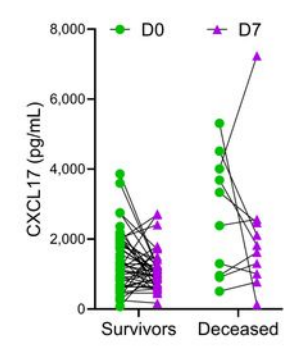

d
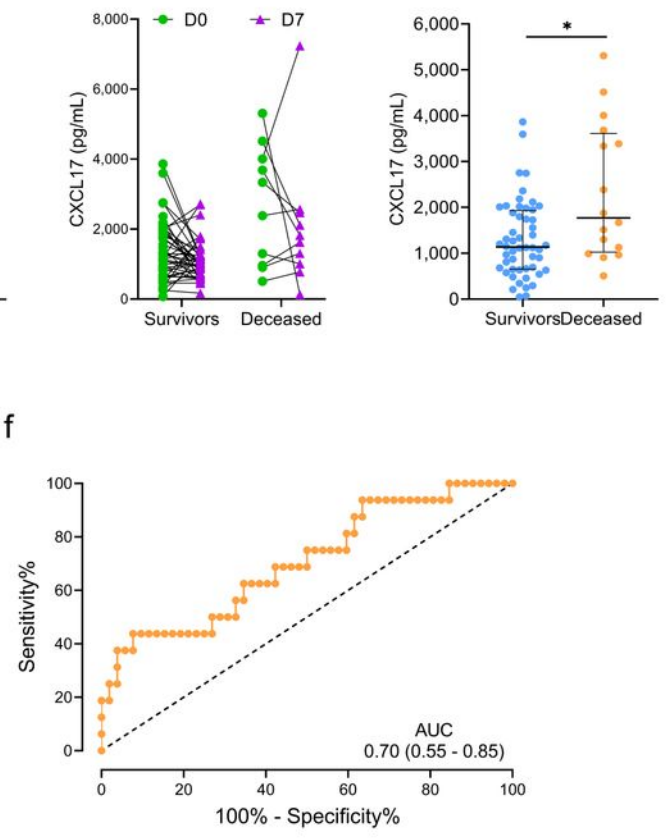

g

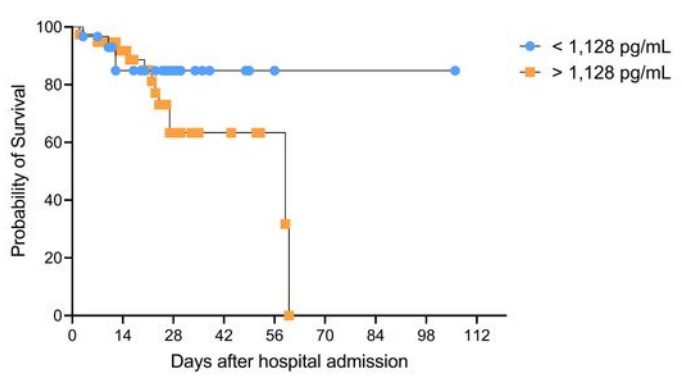

Figure 2

Dynamics and prognostic value of serum CXCL17 levels in influenza (a) Influenza patients were grouped according to their duration of symptoms on admission. Levels of CXCL17 were compared to healthy donors (HD; Kruskal-Wallis test and post hoc Dunn test; graph displays mean CXCL17 levels and the standard error $\left.( \pm S E) ;{ }^{*} \leq \leq 0.05, * * p \leq 0.01, * * * p \leq 0.001, * * * * p \leq 0.0001\right)$. (b) We obtained a second serum sample from influenza subjects seven days (D7) after hospital admission (D0). Levels of CXCL17 at D0 and D7 were compared with the Mann-Whitney U test. (c) Same comparison was performed in survivors and deceased patients (Wilcoxon test in each group). (d) Serum CXCL17 levels in survivor and deceased influenza patients (Mann-Whitney U test; plots display medians and interquartile ranges). (e) Random forest algorithm showing the most important factors that impact on influenza-assocaited mortality. The points represent the mean decrease Gini values, indicative of the importance of each variable with respect to the mean importance of the model (discontinuous vertical line). (f) Receiver operating characteristic (ROC) curve of the levels of CXCL17 in survivor and deceased influenza subjects. The graph displays area under the curve (AUC) and 95\% Cl interval values. (g) Survival curves of influenza patients grouped according to their serum CXCL17 levels were compared with the log-rank test. 
a

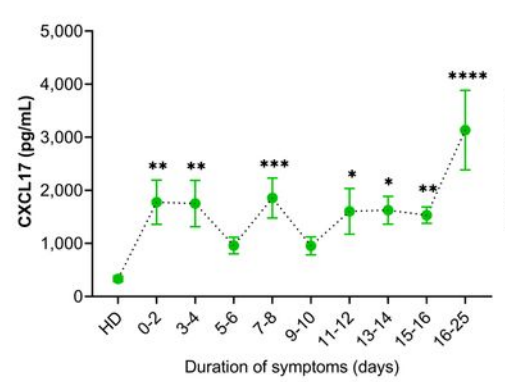

b

e

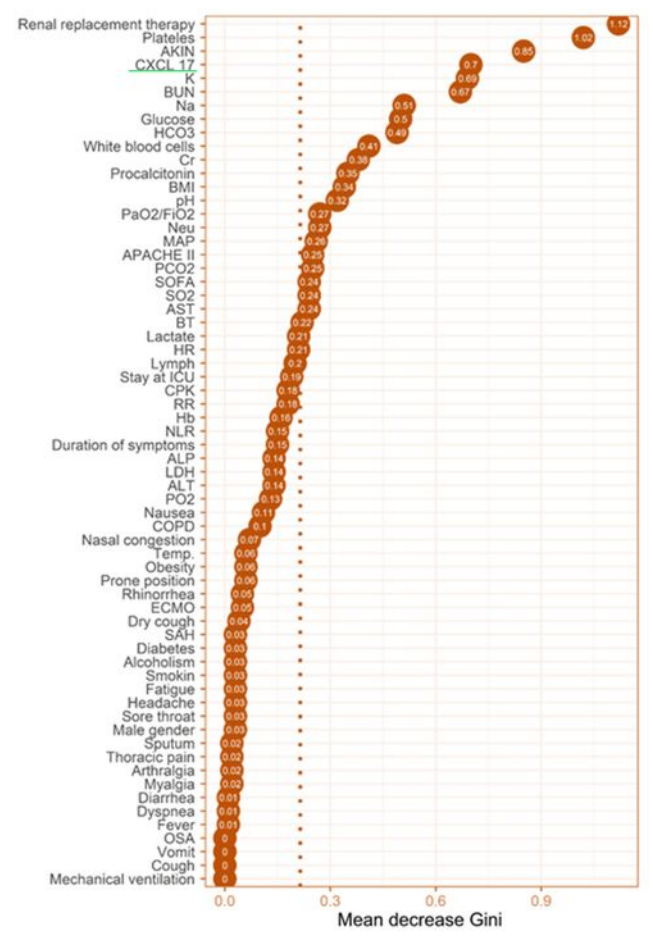

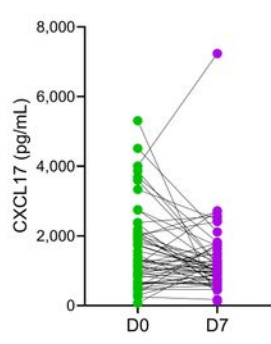
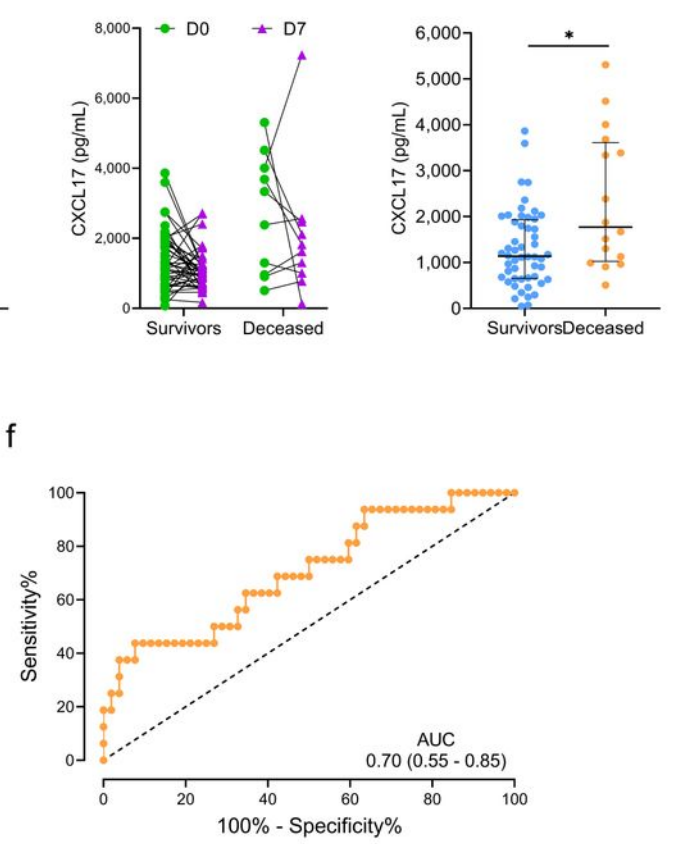

g

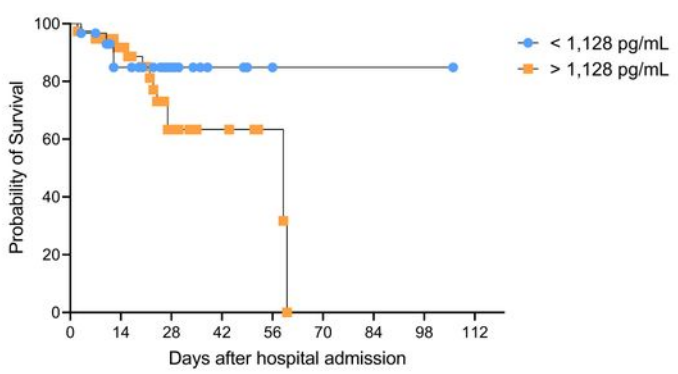

Figure 2

Dynamics and prognostic value of serum CXCL17 levels in influenza (a) Influenza patients were grouped according to their duration of symptoms on admission. Levels of CXCL17 were compared to healthy donors (HD; Kruskal-Wallis test and post hoc Dunn test; graph displays mean CXCL17 levels and the standard error ( \pm SE); $\left.{ }^{\star} p \leq 0.05, * \star p \leq 0.01,{ }^{* \star *} \mathrm{p} \leq 0.001,{ }^{* \star * *} \mathrm{p} \leq 0.0001\right)$. (b) We obtained a second serum sample from influenza subjects seven days (D7) after hospital admission (D0). Levels of CXCL17 at D0 and D7 were compared with the Mann-Whitney U test. (c) Same comparison was performed in survivors and deceased patients (Wilcoxon test in each group). (d) Serum CXCL17 levels in survivor and deceased influenza patients (Mann-Whitney U test; plots display medians and interquartile ranges). (e) Random forest algorithm showing the most important factors that impact on influenza-assocaited mortality. The points represent the mean decrease Gini values, indicative of the importance of each variable with respect to the mean importance of the model (discontinuous vertical line). (f) Receiver operating characteristic (ROC) curve of the levels of CXCL17 in survivor and deceased influenza subjects. The graph displays area under the curve (AUC) and 95\% Cl interval values. (g) Survival curves of influenza patients grouped according to their serum CXCL17 levels were compared with the log-rank test. 
a

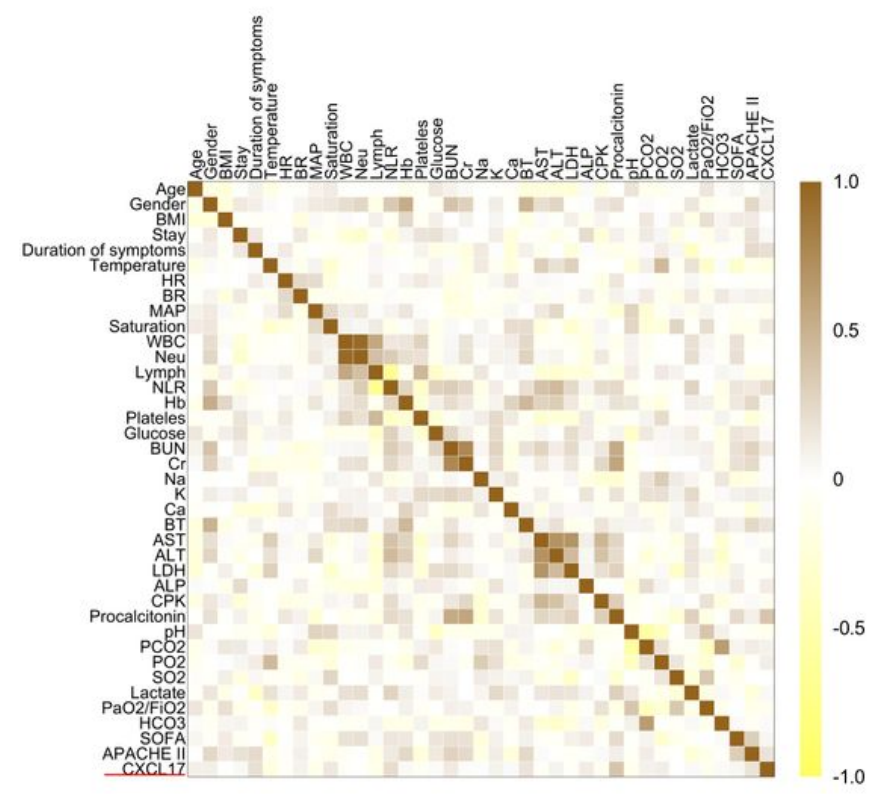

b

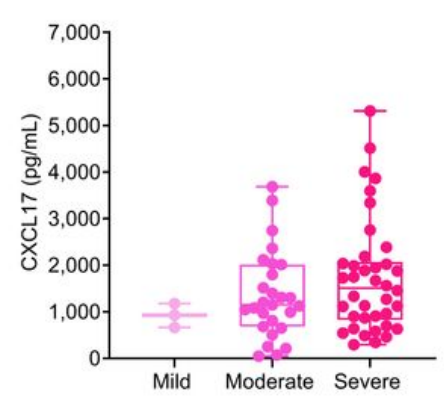

d

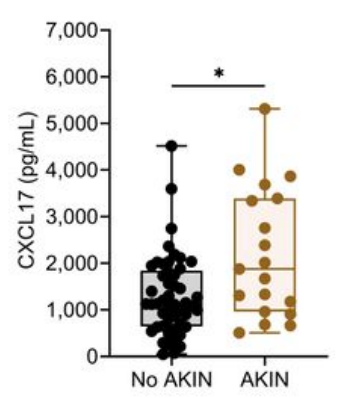

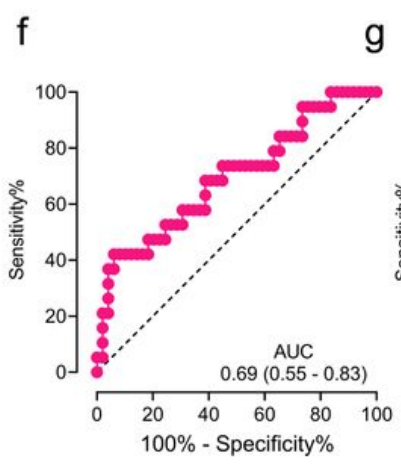

g

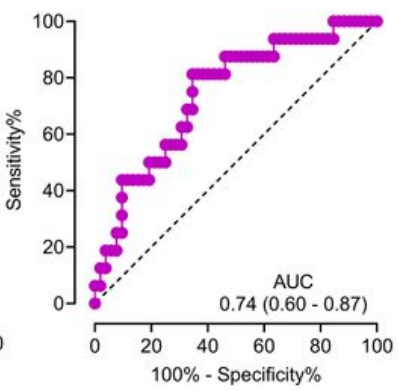

$\mathrm{h}$

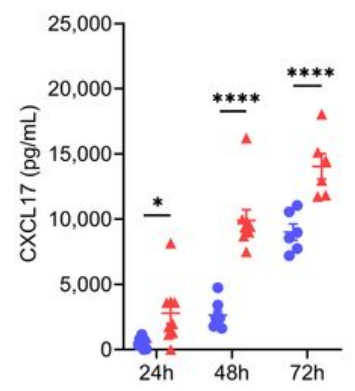

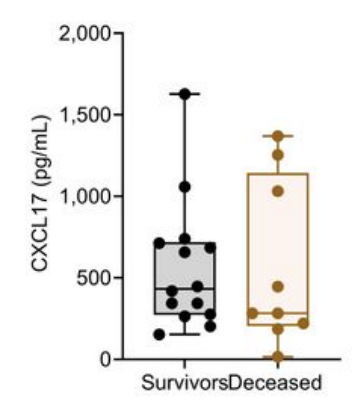

e

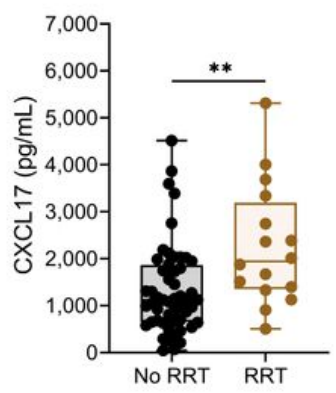

C

i

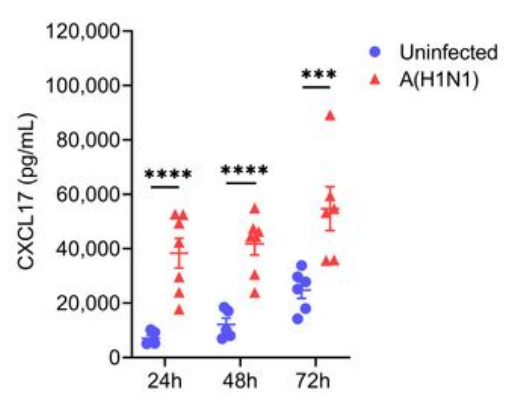

\section{Figure 3}

Extended predictive value of serum CXCL17 levels in influenza but not COVID-19 patients and cellular sources. (a) Multiple correlation analysis of the clinical variables and serum CXCL17 levels of influenza patients. The heat color map gradient was constructed using Spearman R values. (b) Serum CXCL17 levels in influenza patients according to the severity of their acute respiratory distress syndrome (ARDS) on admission (mild, PaO2/FiO2 >200; moderate, $\mathrm{PaO} 2 /$ FiO2 100-200; severe, PaO2/FiO2< 100; Kruskal-Wallis test and post hoc Dunn test). (c) Comparison of the levels of CXCL17 between patients that survived or succumbed to COVID-19. (d) Serum levels of CXCL17 were compared between influenza patients that developed acute kindly injury (AKIN), and individuals with normal renal function. (e) Differences in CXCL17 levels between influenza patients according to their need for renal replacement therapy (RRT). (b-e) Plots display medians and interquartile ranges. (c-e) Mann-Whitney U test. (f) Receiver operating characteristic (ROC) curve of the CXCL17 levels in influenza patients that developed AKIN and those that maintained normal renal function. The graph display area under the curve (AUC) and $95 \% \mathrm{Cl}$ interval values. (g) ROC curve analysis of the serum CXCL17 levels in patients requiring RRT. (h) Human A549 alveolar epithelial cells and (i) peripheral blood-monocyte derived macrophages were infected with a clinical isolate of the influenza A(H1N1) pdm09 virus for 24, 48, and 72h (multiplicity of infection: 5). Levels of CXCL17 in supernatants from infected and uninfected cells were compared using the unpaired Student's $T$ test ( $\mathrm{n}=5$ to 9 per group) at each time point. Values of $\mathrm{p}$ were corrected for multiple comparisons using the Holm-Sidak method. The data represent mean ( \pm SE) values from 2-3 independent experiments per time point and experimental condition. ${ }^{*} p \leq 0.05$, ** $p \leq 0.01, * \star \star p \leq 0.001, * \star \star \star p \leq 0.0001$. 
a

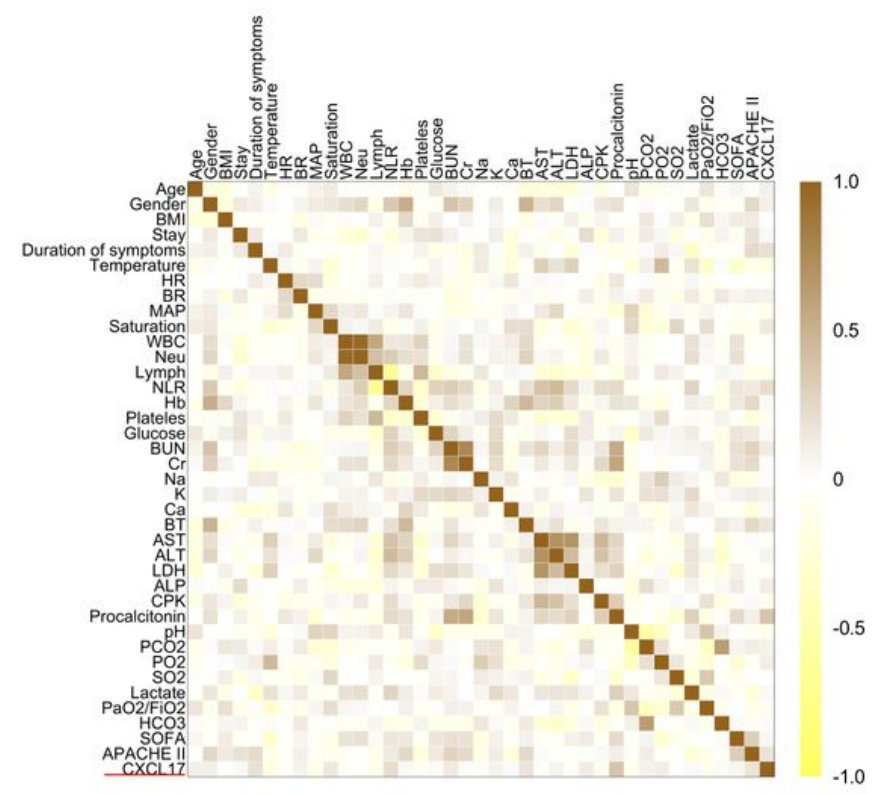

b

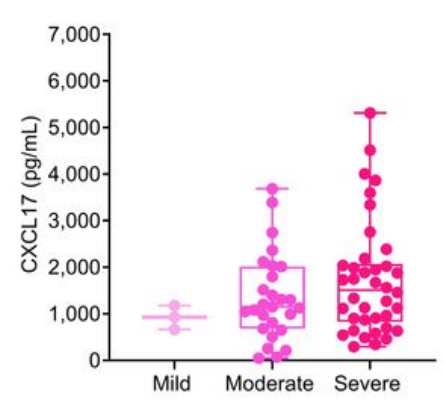

d

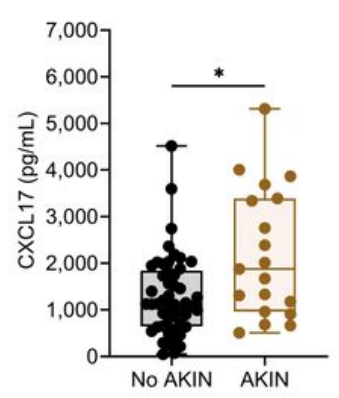

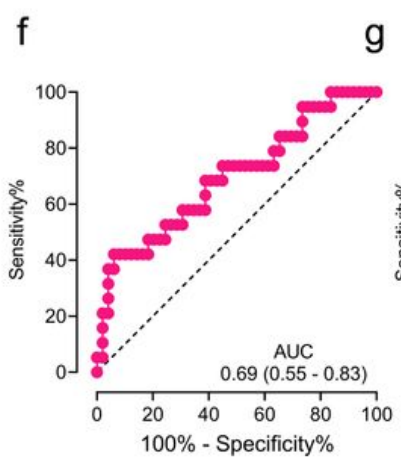

g

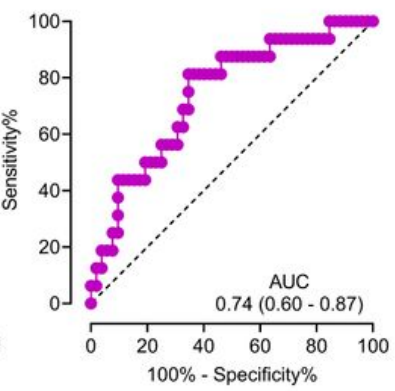

h

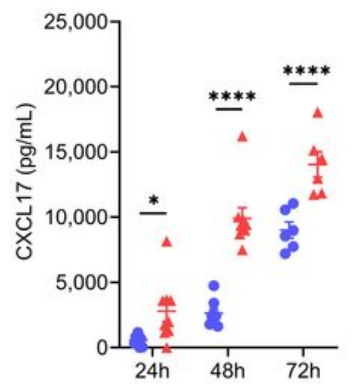

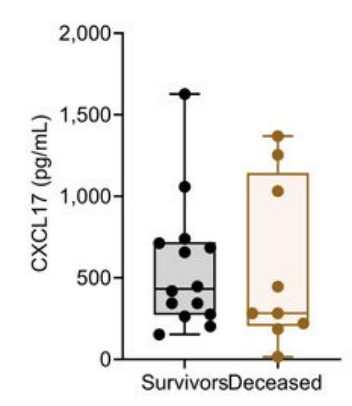

e

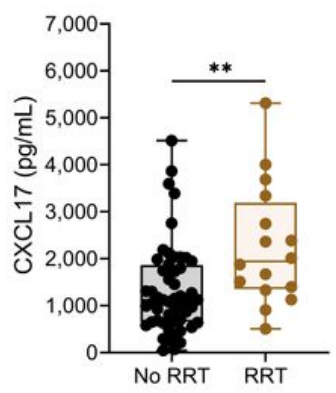

C

i

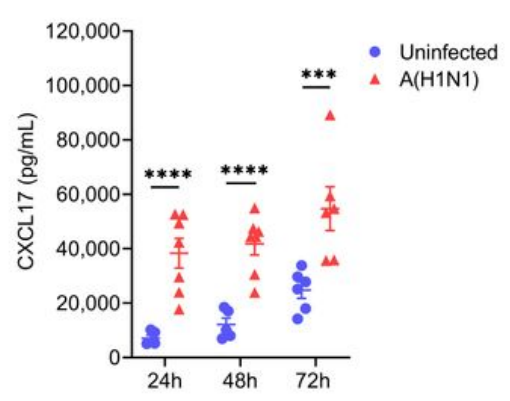

\section{Figure 3}

Extended predictive value of serum CXCL17 levels in influenza but not COVID-19 patients and cellular sources. (a) Multiple correlation analysis of the clinical variables and serum CXCL17 levels of influenza patients. The heat color map gradient was constructed using Spearman R values. (b) Serum CXCL17 levels in influenza patients according to the severity of their acute respiratory distress syndrome (ARDS) on admission (mild, PaO2/FiO2 >200; moderate, $\mathrm{PaO} 2 /$ FiO2 100-200; severe, PaO2/FiO2< 100; Kruskal-Wallis test and post hoc Dunn test). (c) Comparison of the levels of CXCL17 between patients that survived or succumbed to COVID-19. (d) Serum levels of CXCL17 were compared between influenza patients that developed acute kindly injury (AKIN), and individuals with normal renal function. (e) Differences in CXCL17 levels between influenza patients according to their need for renal replacement therapy (RRT). (b-e) Plots display medians and interquartile ranges. (c-e) Mann-Whitney U test. (f) Receiver operating characteristic (ROC) curve of the CXCL17 levels in influenza patients that developed AKIN and those that maintained normal renal function. The graph display area under the curve (AUC) and $95 \% \mathrm{Cl}$ interval values. (g) ROC curve analysis of the serum CXCL17 levels in patients requiring RRT. (h) Human A549 alveolar epithelial cells and (i) peripheral blood-monocyte derived macrophages were infected with a clinical isolate of the influenza A(H1N1) pdm09 virus for 24, 48, and 72h (multiplicity of infection: 5). Levels of CXCL17 in supernatants from infected and uninfected cells were compared using the unpaired Student's $T$ test ( $n=5$ to 9 per group) at each time point. Values of $p$ were corrected for multiple comparisons using the Holm-Sidak method. The data represent mean ( \pm SE) values from 2-3 independent experiments per time point and experimental condition. ${ }^{*} p \leq 0.05$, ** $p \leq 0.01, * \star \star p \leq 0.001, * \star \star \star p \leq 0.0001$. 
a

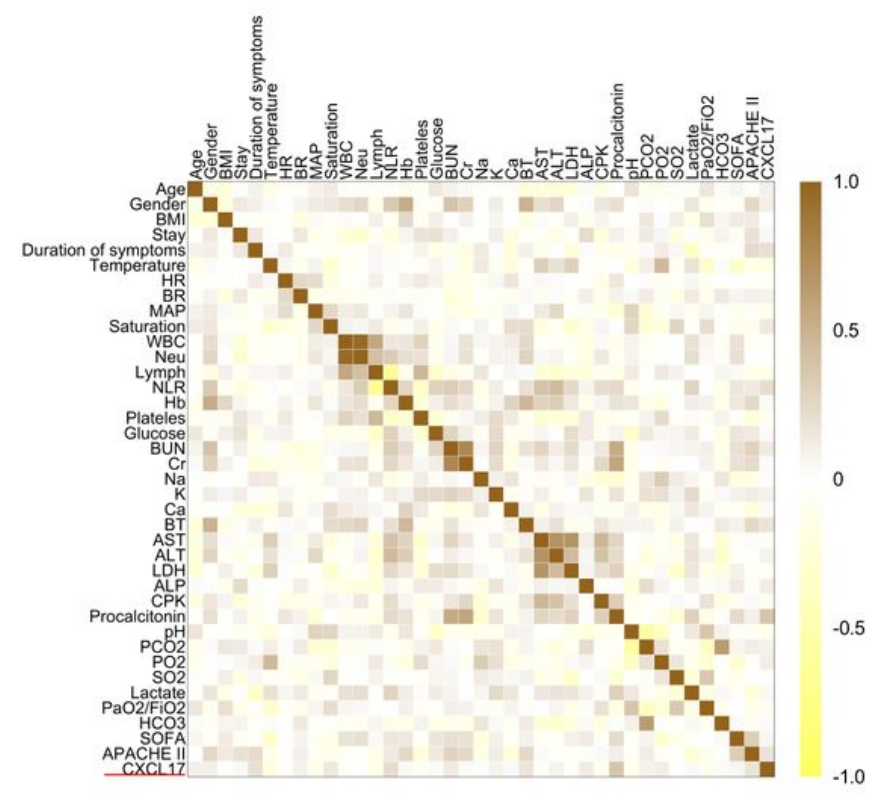

b

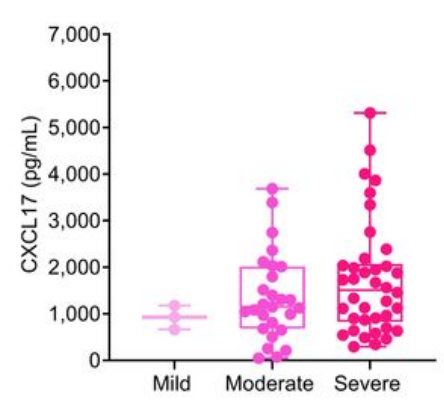

d

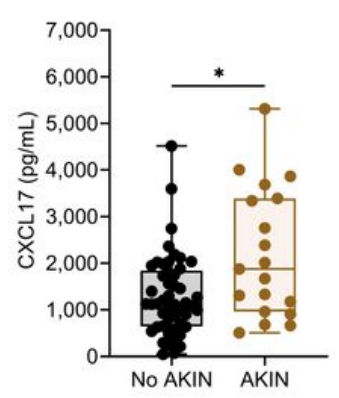

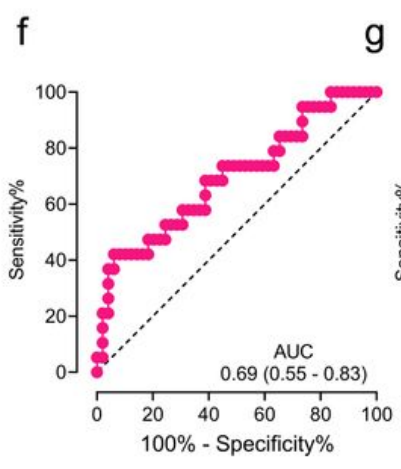

g

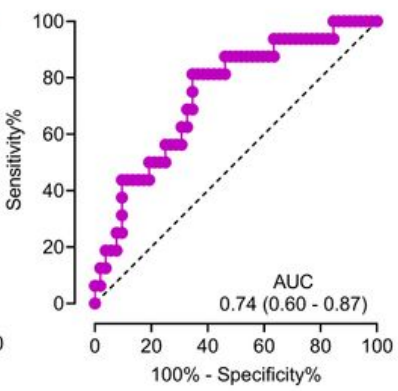

$\mathrm{h}$

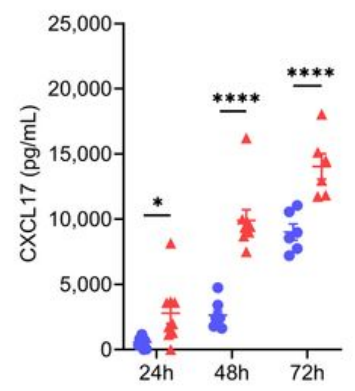

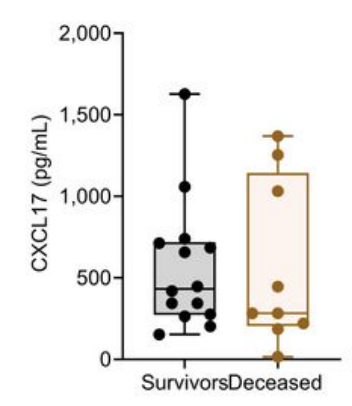

e

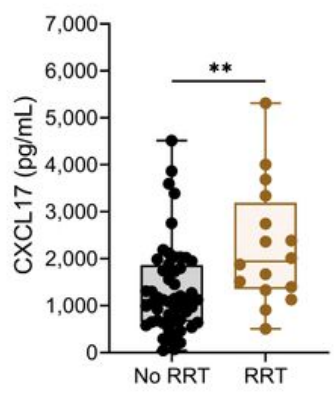

C

i

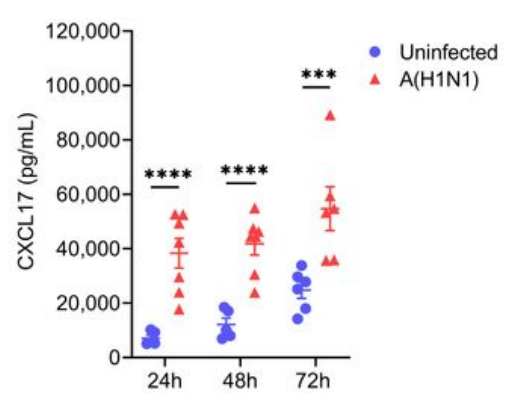

\section{Figure 3}

Extended predictive value of serum CXCL17 levels in influenza but not COVID-19 patients and cellular sources. (a) Multiple correlation analysis of the clinical variables and serum CXCL17 levels of influenza patients. The heat color map gradient was constructed using Spearman R values. (b) Serum CXCL17 levels in influenza patients according to the severity of their acute respiratory distress syndrome (ARDS) on admission (mild, PaO2/FiO2 >200; moderate, PaO2/FiO2 100-200; severe, PaO2/FiO2< 100; Kruskal-Wallis test and post hoc Dunn test). (c) Comparison of the levels of CXCL17 between patients that survived or succumbed to COVID-19. (d) Serum levels of CXCL17 were compared between influenza patients that developed acute kindly injury (AKIN), and individuals with normal renal function. (e) Differences in CXCL17 levels between influenza patients according to their need for renal replacement therapy (RRT). (b-e) Plots display medians and interquartile ranges. (c-e) Mann-Whitney U test. (f) Receiver operating characteristic (ROC) curve of the CXCL17 levels in influenza patients that developed AKIN and those that maintained normal renal function. The graph display area under the curve (AUC) and 95\% Cl interval values. (g) ROC curve analysis of the serum CXCL17 levels in patients requiring RRT. (h) Human A549 alveolar epithelial cells and (i) peripheral blood-monocyte derived macrophages were infected with a clinical isolate of the influenza A(H1N1) pdm09 virus for 24, 48, and 72h (multiplicity of infection: 5). Levels of CXCL17 in supernatants from infected and uninfected cells were compared using the unpaired Student's $T$ test ( $n=5$ to 9 per group) at each time point. Values of $p$ were corrected for multiple comparisons using the Holm-Sidak method. The data represent mean $( \pm S E)$ values from 2-3 independent experiments per time point and experimental condition. ${ }^{*} p \leq 0.05$, ** $p \leq 0.01, * \star * p \leq 0.001, * \star * * p \leq 0.0001$. 
a

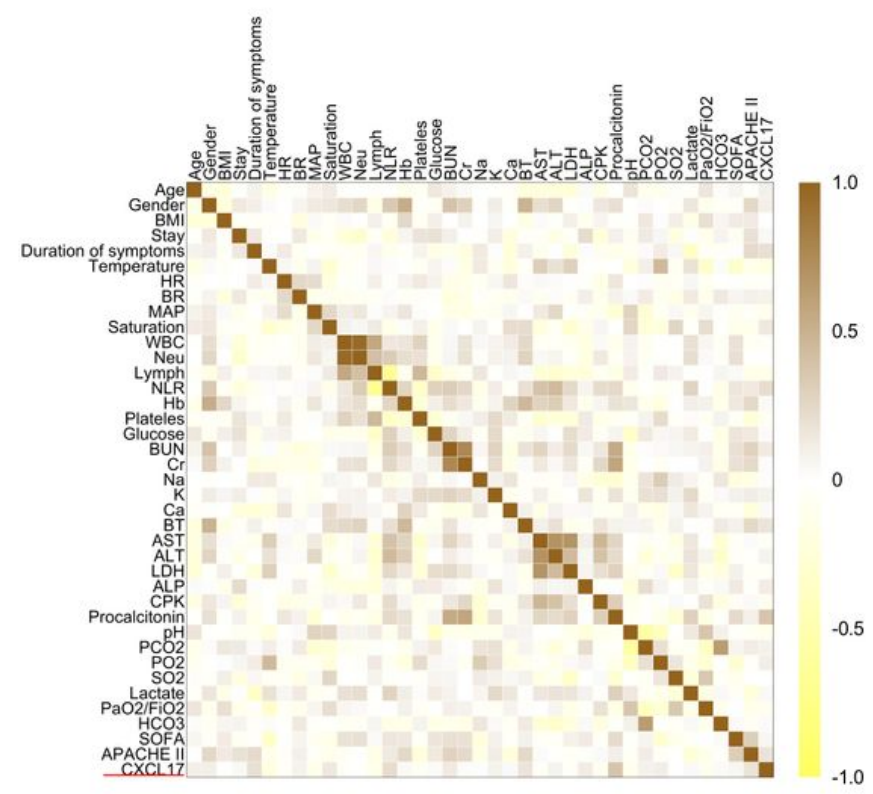

b

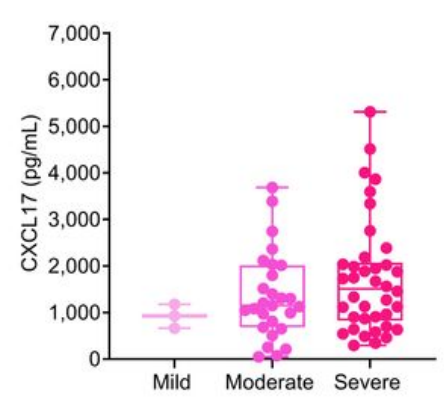

d
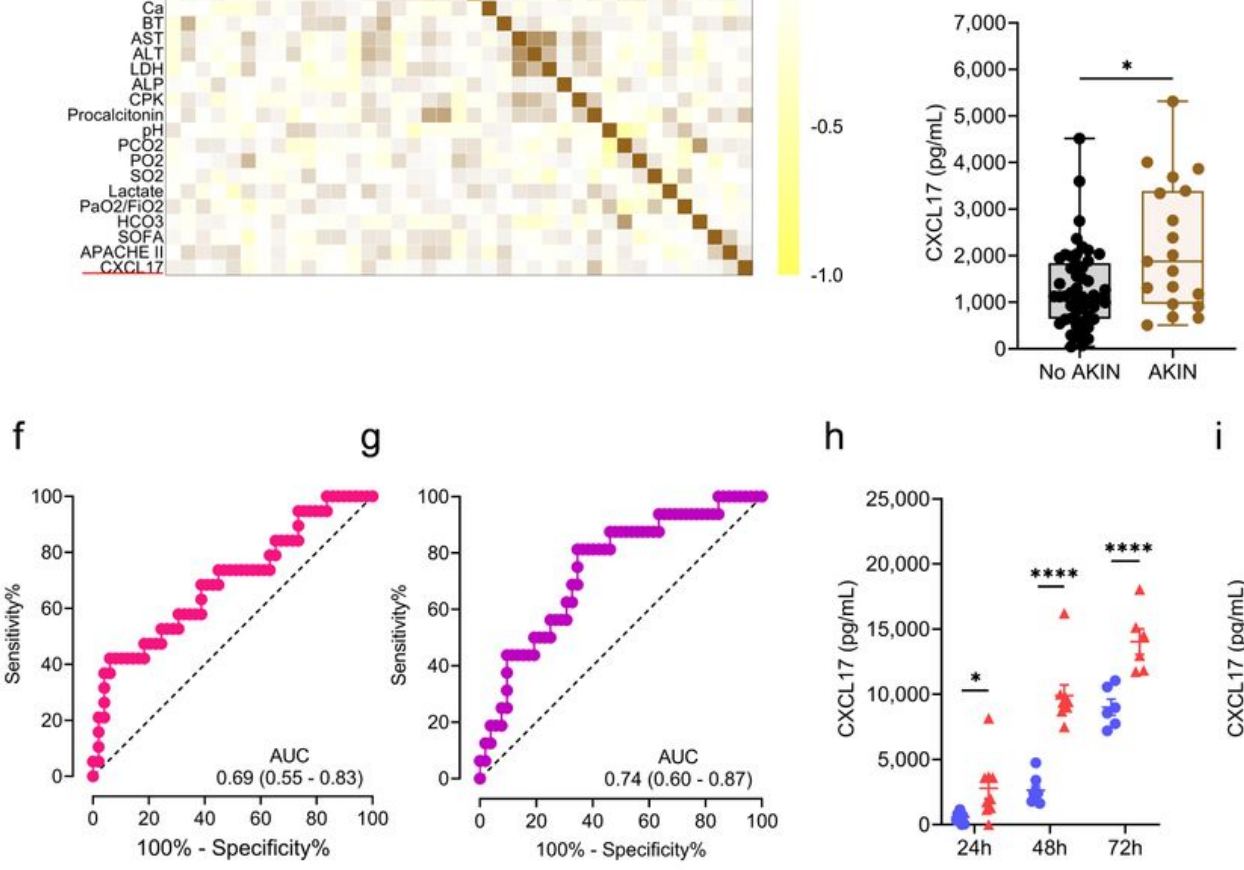

g

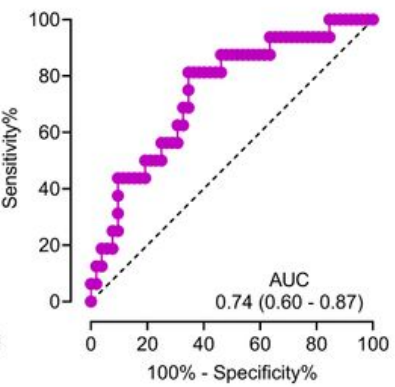

$\mathrm{h}$

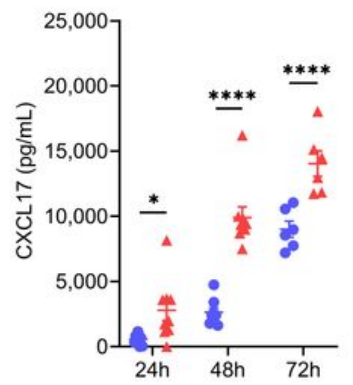

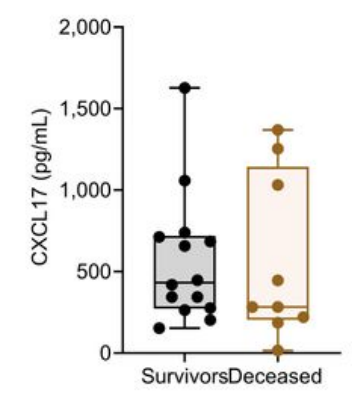

e

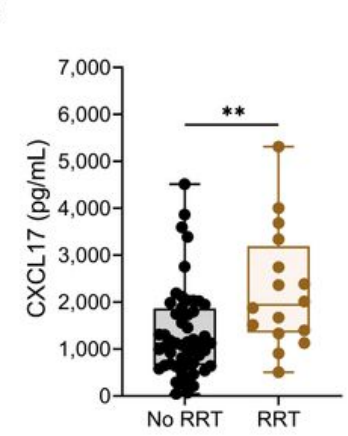

i

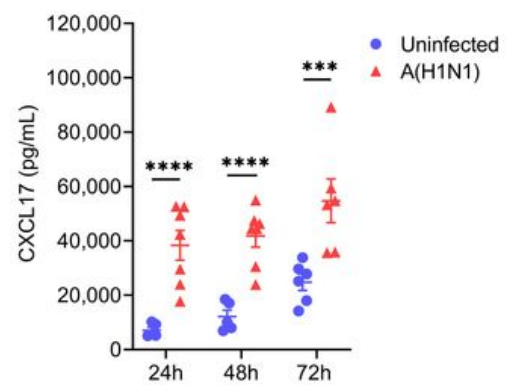

Figure 3

Extended predictive value of serum CXCL17 levels in influenza but not COVID-19 patients and cellular sources. (a) Multiple correlation analysis of the clinical variables and serum CXCL17 levels of influenza patients. The heat color map gradient was constructed using Spearman R values. (b) Serum CXCL17 levels in influenza patients according to the severity of their acute respiratory distress syndrome (ARDS) on admission (mild, PaO2/FiO2 >200; moderate, PaO2/FiO2 100-200; severe, PaO2/FiO2< 100; Kruskal-Wallis test and post hoc Dunn test). (c) Comparison of the levels of CXCL17 between patients that survived or succumbed to COVID-19. (d) Serum levels of CXCL17 were compared between influenza patients that developed acute kindly injury (AKIN), and individuals with normal renal function. (e) Differences in CXCL17 levels between influenza patients according to their need for renal replacement therapy (RRT). (b-e) Plots display medians and interquartile ranges. (c-e) Mann-Whitney U test. (f) Receiver operating characteristic (ROC) curve of the CXCL17 levels in influenza patients that developed AKIN and those that maintained normal renal function. The graph display area under the curve (AUC) and $95 \% \mathrm{Cl}$ interval values. (g) ROC curve analysis of the serum CXCL17 levels in patients requiring RRT. (h) Human A549 alveolar epithelial cells and (i) peripheral blood-monocyte derived macrophages were infected with a clinical isolate of the influenza A(H1N1) pdm09 virus for 24, 48, and 72h (multiplicity of infection: 5). Levels of CXCL17 in supernatants from infected and uninfected cells were compared using the unpaired Student's $T$ test ( $n=5$ to 9 per group) at each time point. Values of $p$ were corrected for multiple comparisons using the Holm-Sidak method. The data represent mean ( \pm SE) values from 2-3 independent experiments per time point and experimental condition. ${ }^{*} p \leq 0.05$, ** $p \leq 0.01, * \star \star p \leq 0.001, * \star \star \star p \leq 0.0001$. 
a

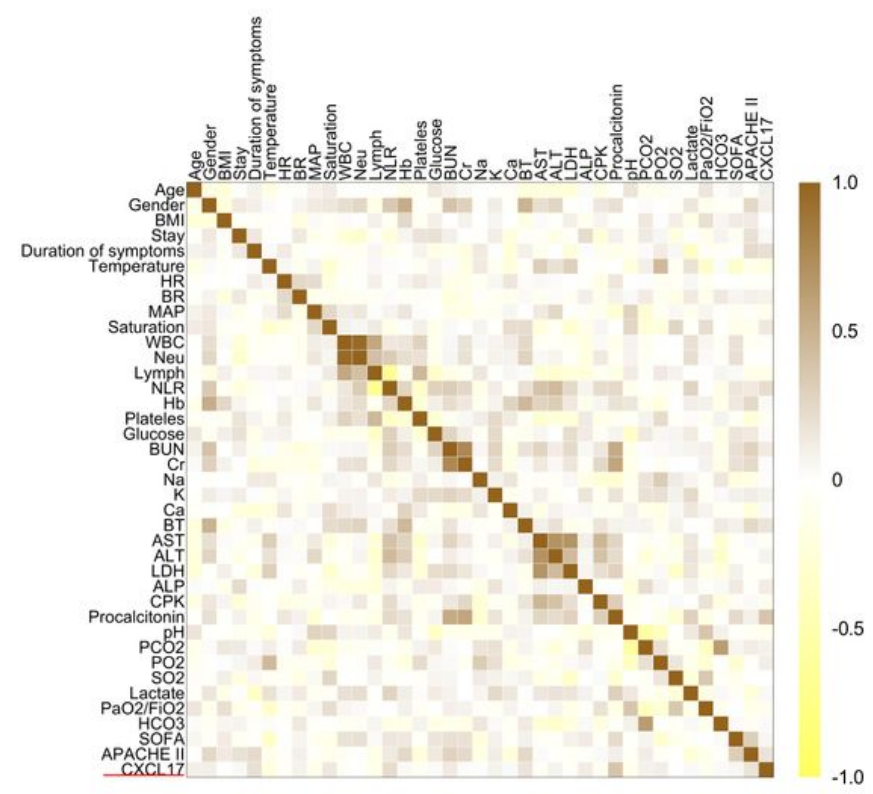

b

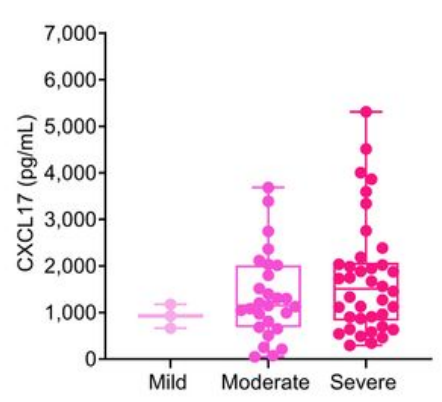

d

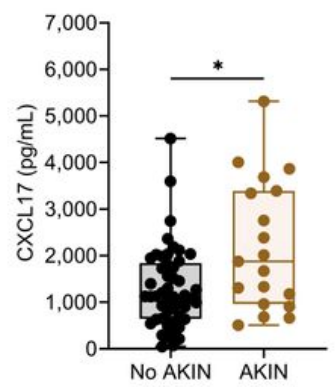

$\mathrm{h}$

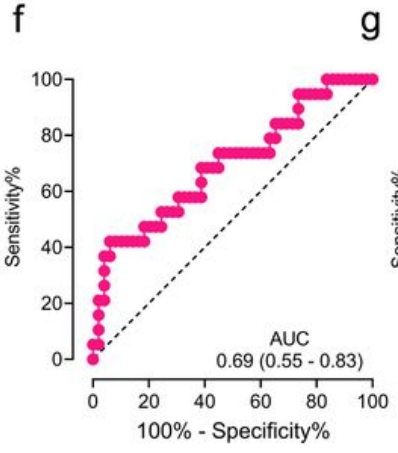

g

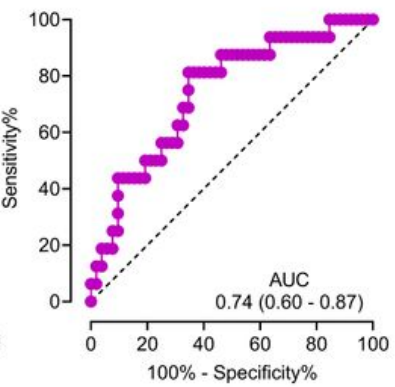

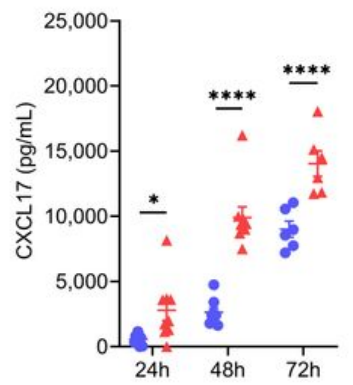

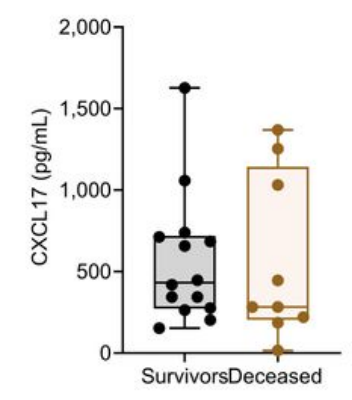

e

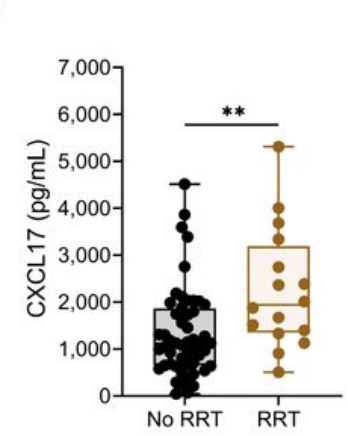

i

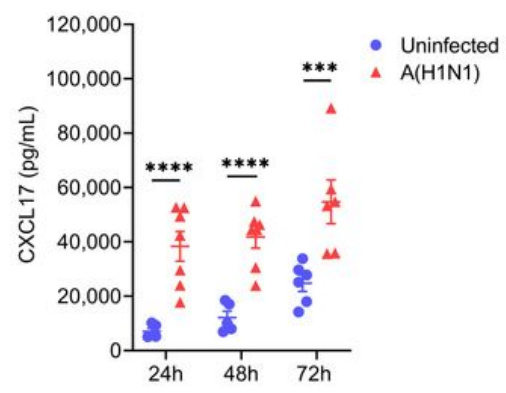

Figure 3

Extended predictive value of serum CXCL17 levels in influenza but not COVID-19 patients and cellular sources. (a) Multiple correlation analysis of the clinical variables and serum CXCL17 levels of influenza patients. The heat color map gradient was constructed using Spearman R values. (b) Serum CXCL17 levels in influenza patients according to the severity of their acute respiratory distress syndrome (ARDS) on admission (mild, PaO2/FiO2 >200; moderate, PaO2/FiO2 100-200; severe, PaO2/FiO2< 100; Kruskal-Wallis test and post hoc Dunn test). (c) Comparison of the levels of CXCL17 between patients that survived or succumbed to COVID-19. (d) Serum levels of CXCL17 were compared between influenza patients that developed acute kindly injury (AKIN), and individuals with normal renal function. (e) Differences in CXCL17 levels between influenza patients according to their need for renal replacement therapy (RRT). (b-e) Plots display medians and interquartile ranges. (c-e) Mann-Whitney U test. (f) Receiver operating characteristic (ROC) curve of the CXCL17 levels in influenza patients that developed AKIN and those that maintained normal renal function. The graph display area under the curve (AUC) and 95\% Cl interval values. (g) ROC curve analysis of the serum CXCL17 levels in patients requiring RRT. (h) Human A549 alveolar epithelial cells and (i) peripheral blood-monocyte derived macrophages were infected with a clinical isolate of the influenza A(H1N1) pdm09 virus for 24, 48, and 72h (multiplicity of infection: 5). Levels of CXCL17 in supernatants from infected and uninfected cells were compared using the unpaired Student's $T$ test ( $n=5$ to 9 per group) at each time point. Values of $p$ were corrected for multiple comparisons using the Holm-Sidak method. The data represent mean ( \pm SE) values from 2-3 independent experiments per time point and experimental condition. ${ }^{*} p \leq 0.05$, ** $p \leq 0.01, * \star \star p \leq 0.001, \star \star \star \star x \leq 0.0001$.

\section{Supplementary Files}


This is a list of supplementary files associated with this preprint. Click to download.

- SupplementalData.docx

- SupplementalData.docx

- SupplementalData.docx

- SupplementalData.docx

- SupplementalData.docx 